\title{
The VLA Low-frequency Sky Survey
}

\author{
A. S. Cohen ${ }^{1}$, W. M. Lane ${ }^{1}$, W. D. Cotton ${ }^{2}$, N. E. Kassim ${ }^{1}$, T. J. W. Lazio ${ }^{1}$, \\ R. A. Perley ${ }^{3}$, J. J. Condon ${ }^{2}$, W. C. Erickson ${ }^{4}$,
}

\begin{abstract}
The Very Large Array (VLA) Low-frequency Sky Survey (VLSS) has imaged $95 \%$ of the $3 \pi$ sr of sky north of $\delta=-30^{\circ}$ at a frequency of $74 \mathrm{MHz}$ (4 meter wavelength). The resolution is $80^{\prime \prime}$ (FWHM) throughout, and the typical RMS noise level is $\langle\sigma\rangle \approx 0.1 \mathrm{Jy} /$ beam. The typical point-source detection limit is $0.7 \mathrm{Jy} /$ beam and so far nearly 70,000 sources have been catalogued. This survey used the $74 \mathrm{MHz}$ system added to the VLA in 1998. It required new imaging algorithms to remove the large ionospheric distortions at this very low frequency throughout the entire $\sim 11.9^{\circ}$ field of view. This paper describes the observation and data reduction methods used for the VLSS and presents the survey images and source catalog. All of the calibrated images and the source catalog are available online (URL=http://lwa.nrl.navy.mil/VLSS) for use by the astronomical community.
\end{abstract}

Subject headings: surveys — catalogs — atmospheric effects — radio continuum: general

\section{Introduction}

Recently, increasingly powerful telescopes and data-reduction abilities have made it possible to complete comprehensive and sensitive radio surveys, notably the $325 \mathrm{MHz}$ Westerbork Northern Sky Survey (WENSS; Rengelink et al. 1997), the 843 MHz Sydney University Molonglo Sky Survey (SUMSS; Bock et al. 1999), the 1.4 GHz NRAO VLA Sky Survey (NVSS; Condon et al. 1998) and the 1.4 GHz Faint Images of the Radio Sky at Twenty-cm

\footnotetext{
${ }^{1}$ Naval Research Laboratory, Code 7213, Washington, DC, 20375 USA, Aaron.Cohen@nrl.navy.mil, Wendy.Lane@nrl.navy.mil

${ }^{2}$ National Radio Astronomy Observatory, 520 Edgemont Road, Charlottesville, VA, 22903 USA

${ }^{3}$ National Radio Astronomy Observatory, P.O. Box 0, Socorro, NM 87801 USA

${ }^{4}$ School of Mathematics and Physics, University of Tasmania, Hobart, TAS 7005, Australia
} 
survey (FIRST; Becker et al. 1995; White et al. 1997). These four surveys have resulted in the detection of millions of radio sources. Their data have already made a valuable contribution to topics such as the nature of extragalactic radio sources and their relation to galaxy formation, the large-scale structure of the universe, and the use of Galactic foreground polarization as a probe of the interstellar medium in our own Galaxy. In addition, the simple web access to maps and source information of the NVSS and FIRST databases has made it easy for researchers with little or no experience observing at radio frequencies to use this information in their work.

Until recently, it was impossible to make images approaching the dynamic range and angular resolution of WENSS, SUMSS, and the NVSS at frequencies below $150 \mathrm{MHz}$ owing to the severe ionospheric phase changes at such low frequencies. This changed with the development of a $74 \mathrm{MHz}$ (4-meter wavelength) system on the VLA (Kassim et al. 1993), which enabled sub-arcminute resolution synthesis imaging with a connected-element interferometer below $150 \mathrm{MHz}$ for the first time. Fully implemented in 1998, this new system has produced interesting science and provided valuable experience in the challenges associated with low-frequency observing at high angular resolution. For a complete description of the VLA $74 \mathrm{MHz}$ system and its capabilities, see Kassim et al. (2007).

Phase distortions from the ionosphere and a large field of view introduce a particularly difficult problem for high-resolution imaging at $74 \mathrm{MHz}$. The isoplanatic patch is defined as a region on the sky small enough that angular variations in the ionospheric phase distortions across it are negligible. Unlike at higher frequencies, the isoplanatic patch at $74 \mathrm{MHz}$ for the VLA A and B-configurations (with maximum baselines of 36 and $11 \mathrm{~km}$ respectively) is significantly smaller than the field of view. Therefore, initially the only sources that could be imaged were those strong enough that all other sources in the field of view outside of its isoplanatic patch were weak enough in comparison to be ignored during calibration. This restricted the system to sources with flux densities of at least $\sim 100 \mathrm{Jy}$ at $74 \mathrm{MHz}$. This obstacle has recently been greatly reduced through the development of new calibration algorithms (described in Section 4) and the availability of the necessary computational power to implement them. It is now possible to image an entire field of view and detect sources as weak as $\sim 0.1$ Jy during most circumstances for the $11 \mathrm{~km}$ B-configuration and in many cases for the $35 \mathrm{~km}$ A-configuration.

These new algorithms have greatly extended the scientific capability of the $74 \mathrm{MHz}$ VLA system, and have made it possible to conduct efficient surveys. In 2002 we began the VLA Low-frequency Sky Survey (VLSS), a $74 \mathrm{MHz}$ survey of the entire sky north of $\delta>-30^{\circ}$. VLSS images have a resolution of $80^{\prime \prime}$ and a median map RMS noise level of $\sim 0.1 \mathrm{Jy} /$ beam. The specific survey parameters are summarized in Table 1. This paper 
describes the methods and presents the results (images and a source catalog) of the VLSS, which is now $95 \%$ complete. In a future paper we will present analysis of these results and their scientific implications.

\section{Survey Motivation}

There are several scientific motivations for a new, relatively high resolution, high sensitivity survey at $74 \mathrm{MHz}$. The first is the study of ultra-steep-spectrum objects $(\alpha<$ -1.3). These include halos and relics in clusters of galaxies (Enßlin \& Röttgering 2002), fossil radio galaxies (Slee et al. 2001), high redshift radio galaxies (Chambers et al. 1987; De Breuck et al. 2000) and pulsars. The VLSS has the potential not only to help study known examples of such objects, but also to help in the discovery of new objects in these classes. The second main advantage of a $74 \mathrm{MHz}$ survey is that the spectra of known objects can be extended to a frequency low enough that extrinsic (eg. free-free absorption) and intrinsic (eg. synchrotron self-absorption, electron energy-spectral cutoffs, free-free absorption) spectral effects can be distinguished (Kassim et al. 1995). This is useful for studying physical processes related to acceleration, turbulence, and propagation in normal galaxies, supernova remnants, HII regions, and the interstellar medium. Third, extragalactic samples selected at $74 \mathrm{MHz}$ are dominated by isotropic (lobe-dominated) radio emission, unlike those found at higher frequencies. A $74 \mathrm{MHz}$ sample thus provides an unbiased view of the parent populations used in "unification" models to account for the diverse source populations observed at higher frequencies (eg. Wall \& Jackson 1997). Finally, a large survey that pushes the phase space of previous observations is often useful in uncovering rare, but potentially important new phenomena.

On a technical level, the VLSS will produce a low-frequency sky model and an initial calibration grid for future low-frequency telescopes, such as the Low Frequency Array (LOFAR) and the Long Wavelength Array (LWA). The VLSS catalog will also help in the design of future low-frequency telescopes since the still-unsolved problem of calibrating at low frequencies on long baselines $(>50 \mathrm{~km}$ ) will likely depend on the expected number of calibrator sources available within a field of view.

\section{Observation Strategy}

Observations were carried out in four observational programs over the course of five years (see Table 21). They are substantially complete at this time; 10 hours remain in each 
of the upcoming B- and BnA-configurations to re-observe a few remaining high-noise fields. Overall we have used just over 900 hours of VLA time for this survey. During each phase of observations every effort was made to observe contiguous sky areas so that the intermediate catalog releases would be as useful as possible for science.

\subsection{Pointing Grid}

The smallest element of the VLSS is the image of a single field of view surrounding a given pointing center. The primary-beam sensitivity pattern of a 25-meter VLA dish at $74 \mathrm{MHz}$ is a circular Gaussian with a full-width at half maximum (FWHM) diameter of $11.9^{\circ}$. In order to obtain uniform sensitivity over the entire survey area, overlapping observations were made on a roughly hexagonal grid of 523 pointing centers covering the entire sky north of $\delta>-30^{\circ}$. The grid spacing was

$$
\Delta \approx \theta_{p} / \sqrt{2} \approx 8.6^{\circ}
$$

where $\theta_{p}$ is the FWHM primary beamwidth. The partially overlapping images of each field are weighted to correct for the primary-beam attenuation and combined to produce the final

sky images as described by Condon et al. (1998). For the same RMS noise level at the center of each pointing, this grid produces nearly uniform sensitivity (Figure 1).

\subsection{Resolution}

Fields in the declination range $-10^{\circ}<\delta<80^{\circ}$ were observed in the B-configuration which produces a dirty-beam size of between $60^{\prime \prime}$ and $80^{\prime \prime}$ at this frequency. In order to produce uniform resolution and avoid highly elongated beams for fields that never reach high elevations, all fields at declinations $\delta>80^{\circ}$ and $\delta<-10^{\circ}$ were observed in the BnAconfiguration which provides much longer baselines along the north-south axis than along the east-west axis. All images were restored with a circular 80" FWHM beam for uniform resolution throughout the entire survey region.

\section{3. $\quad 74 \mathrm{MHz}$ Specific Considerations}

The observations had a bandwidth of $1.56 \mathrm{MHz}$ centered on the radio astronomy allocation of $73.8 \mathrm{MHz}$. In order to facilitate the excision of radio-frequency interference (RFI) and minimize bandwidth smearing, we observed in multi-channel continuum mode with 128 
channels after online Hanning smoothing. We used an integration time of 10 seconds, the smallest available on this system, which tangentially convolves the point-source response by a function whose width is proportional to the distance from the phase center and reaches $15^{\prime \prime}$ at the primary half-power circle. Convolved with the $80^{\prime \prime}$ restoring beam, this yields a point-source response that is extended to $81.4^{\prime \prime}$ in the tangential direction at the primary half-power point.

Ionospheric distortions increase with decreased elevation. Therefore we required that all fields in the B-configuration be observed at elevations $\geq 30^{\circ}$, and the lower-declination BnA-configuration fields were observed at elevations $\geq 20^{\circ}$. In all cases efforts were made to observe fields at the highest elevations possible.

At $74 \mathrm{MHz}$ scattering in the solar-wind plasma distorts radio sources viewed through it. In order to minimize this distortion, all observations were made at solar elongations $\geq 60^{\circ}$. In addition, observations were scheduled during night-time as much as possible, because the ionosphere is less stable during the daytime, and particularly the morning.

\subsection{Time per Pointing}

We integrated a minimum of 75 minutes on each field in order to reach our survey sensitivity goal of $100 \mathrm{mJy} /$ beam average RMS noise level at the field centers. In order to improve our spatial frequency coverage, each field was observed at multiple hour angles by dividing the total 75 minutes on source into three shorter observations, each about 25 minutes, that were separated in time at least one hour.

We used Cygnus A (3C 405) as the sole bandpass and complex-gain calibrator for all observations. When the elevation of Cygnus A was not high enough, we observed Virgo A and 3C 123 instead with the intent of using them as calibrators. Experience showed, however, that the bandpasses and instrumental gains were stable (to within a few percent) over periods of as long as two days. As a result, using calibration gains derived from the nearest Cygnus A observation in time (up to two days earlier or later) proved more reliable in our pipeline reduction than trying to use the two weaker calibrator sources. Therefore, neither Virgo A nor 3C 123 was ever used as a calibrator. We typically observed a calibrator for three to five minutes once every two hours. We did not observe any secondary or phase calibrators for two main reasons. First, because of the large primary beam size and low antenna gain at $74 \mathrm{MHz}$ there are only a few sources in the entire sky that would be suitable. Second, because of the angular structure of the ionospheric fluctuations, gains calculated more than a few degrees away from the target source are not useful. 
We planned 1.5 hours of time per pointing center to accommodate the 75 minutes integration on source, slewing between fields, and calibration scans. The slew times necessarily varied from observation to observation; when available, extra time was used to integrate longer and/or increase the number of scans on each pointing. Typically, observations were made in 6-20 hour blocks.

\subsection{Re-Observations}

For about $65 \%$ of the fields, the above observation strategy was sufficient to produce maps that met our survey criteria. However, some fields had unacceptably high RMS noise levels after 75 minutes of integration. The principal causes of high map noise were RFI or strong ionospheric turbulence, which can render unusable some fraction of the observing time on a given field. We have re-observed most of these fields with additional 20-25 minute scans. The new data were then combined with the old and a new map (usually with lower noise) was produced. If that map still had high noise levels, more time was scheduled. Including time for calibration and slewing, nearly $20 \%$ of our total project time was used for re-observations of this nature.

\section{Data Reduction Method}

\subsection{Calibration}

\subsubsection{Cygnus $A$ as a Calibrator}

VLSS data were calibrated using the radio galaxy Cygnus A (3C 405). Its 17,000 Jy is by far the highest flux density for any non-variable object in the sky at $74 \mathrm{MHz}$. (Cassiopeia A has a similar flux density, but this is not constant in time, and it is a much more extended source and therefore is fainter on the longer baselines.) This high flux density is crucial because to function as a calibrator, a source must dominate the total flux density in its field of view, and at $74 \mathrm{MHz}$ the $11.9^{\circ}$ field typically contains several hundred Jy of flux density in background sources. Another advantage of such high flux density is that even in narrow $(12 \mathrm{kHz})$ channels the source is nearly always far stronger than any RFI. Because of its relatively high declination, Cygnus $\mathrm{A}$ is at an elevation of at least $30^{\circ}$ for over 10 hours per day at the VLA, a time period which nearly always overlapped with some portion of our observing sessions. For the few observations where it was never at high enough elevation, a scan from the day before or day after was used, as this was more reliable than using a weaker 
source and kept the flux scale consistent. We observed Cygnus A for roughly 3 minutes every 2 hours or so while it was above an elevation of $30^{\circ}$. Even at our resolution of $80^{\prime \prime}$, Cygnus A is heavily resolved, and we used a pre-existing image as a calibration model (available from http://lwa.nrl.navy.mil/tutorial). This model has been scaled to have the same total flux density as calculated using the spectral model from Baars et al. (1977), which is 17,086 Jy at $73.8 \mathrm{MHz}$. This defines the flux-density scale of the entire survey. The accuracy and reliability of this flux-density scale will be discussed in further detail in Section 7.3.2.

\subsubsection{Bandpass and Amplitude Gain Calibration}

As our observations were conducted in spectral-line mode, it was necessary to perform a bandpass calibration. This was done for each observation cycle using the existing scans of Cygnus A and using the pre-existing Cygnus A model. A few channels at the center, known to be generally free of RFI, were used to normalize the bandpass and set the zero-point for the phases. The resulting bandpass solutions were then inspected by hand. Occasionally one or more antennas were not functional, and this could immediately be identified as a bandpass that appeared pathologically shaped or that was simply random noise. Once identified, the data from these antennas were flagged from all sources for these times and the bandpasses for all antennas were re-calculated.

Next we performed a gain calibration, again by comparing the scans of Cygnus A to its pre-existing model. This again provided an opportunity to remove defective data. We removed data showing amplitude solutions with a scatter among adjacent time intervals that was much greater than normal. Also, typical amplitude gains at $74 \mathrm{MHz}$ vary over time by about $10 \%$ or less, and so, if an antenna varied by much more than this, it was also flagged. As we could only see these solutions during the times we observed Cygnus A, we removed all data surrounding a "bad" scan on Cygnus A.

The gain calibration produced reliable amplitude gains; however, the gain phases depend greatly on the location in the sky and therefore could not be transfered from Cygnus A to

any given field. Determination of the gain phases toward the field of interest would be done at a later stage.

\subsubsection{Instrumental Phase Gains}

Although we could not fully determine the phase gains simply by calibrating to Cygnus A, it was necessary to estimate the instrumental contributions to the phase as they are not cor- 
rected by the technique described later. For any baseline, the observed phase is the sum of four components:

$$
\phi=\phi_{\text {src }}+\phi_{\text {inst }}+\phi_{\text {ion }}+\phi_{\text {noise }}
$$

where $\phi_{s r c}$ is the phase contributed by the structure of the source, $\phi_{\text {inst }}$ is the phase contributed by the VLA instruments, $\phi_{\text {ion }}$ is the phase produced by the ionosphere and $\phi_{\text {noise }}$ is due to the noise from the Galactic background and the thermal noise in the electronics.

Observations of Cygnus A can be used to isolate these components and obtain an estimate of $\phi_{\text {inst }}$. This is because Cygnus A is such a strong source that it completely dominates its field of view and therefore the overwhelming contribution to $\phi_{\text {ion }}$ comes from a single isoplanatic patch. The first step in determining $\phi_{\text {inst }}$ was to observe Cygnus A in a number of scans separated in time. A standard phase-calibration procedure was used to estimate the antenna-based phases every 10 to 30 seconds and a model of Cygnus A was used to separate this from the phases produced by the source morphology, $\phi_{s r c}$. Since $\phi_{\text {noise }}$ is assumed to be small and uncorrelated in time, it is assumed to average out to a negligible level over the course of the several scans on Cygnus A and can be ignored in the following. This leaves the antenna based $\phi_{\text {inst }}$ and $\phi_{i o n}$ terms in the calibration results. The ionospheric phase is the sum of a linear gradient across the array and higher-order terms. The linear gradient causes a position shift and the higher-order terms defocus the array. It is not possible to uniquely determine a set of instrumental phases as it is not possible to distinguish among the set of instrumental phases plus a linear gradient across the array. However, since the data calibration procedure described later can determine and correct linear gradients, a set of instrumental phases plus an arbitrary linear gradient is sufficient.

A reference time segment of well-behaved data is used to define the instrumental plus linear phase gradient. A calibration at a single time at which there were no higher order ionospheric phase terms would be sufficient for calibration; however, this is infrequent and cannot be established from measurements at a single time. Thus, a least squares procedure is used in which the time sequence of calibrator results are used to fit:

\section{Instrumental phase}

per antenna and receiver (plus an arbitrary linear gradient).

\section{Linear Gradient}

across the array for each calibration time with respect to the reference time interval.

The higher-order ionospheric phase terms are assumed uncorrelated over the time range of the calibration data implying that their influence will average out. Residuals from the fitting can be used to determine when the ionosphere is too disturbed and the higher-order 
terms dominate, as well as the occasional phase jumps in the VLA electronics. Periods of overly disturbed ionospheric conditions were flagged and the calibration procedure repeated. Occasionally phase jumps were discovered in the data and they were corrected and the calibration procedure repeated. Once the instrumental phases are used to correct the data, it is possible to use the data to image the sky, albeit with a time and position dependent systematic position offset.

\subsection{RFI Excision}

\subsubsection{Need for Automated Procedure}

RFI causes excess signal to appear in the visibility data. The best way to remove this is by looking at plots of phase or amplitude as a function of time and frequency channel and removing contaminated data by hand (Lane et al. 2005). However, with 523 fields, each with 120 channels, 351 baselines and both right and left circular polarizations, there was no practical way to perform this type of flagging by hand. Therefore flagging of data contaminated with RFI was done using automated procedures.

\subsubsection{Removing Bad Channels}

The first step in flagging was to remove channels which are known to nearly always be contaminated with internally produced RFI. These channels are nearly equally spaced across the bandpass (seen in Figure 2). This internally generated interference is often seen in $74 \mathrm{MHz}$ VLA data and comes from the VLA DCS system (used to send command and control data around the array) and results in a well-known "100 kHz comb" of narrow-band harmonics distributed across the bandpass.

\subsubsection{Clipping Ultra-High Visibilities}

The next step in our automated RFI-flagging procedure was to "clip" all visibilities with amplitudes above a flux level greater than what could conceivably come from astronomical sources in the field of view. The clipping threshold was determined automatically using an algorithm that fit the existing $u v$-data to solve for the zero-spacing flux density (ie. total flux density in the field of view). The total flux density in a field of view had a median value of about $330 \mathrm{Jy}$ for all fields we observed, but of course certain fields with unusually strong 
sources had much higher total flux density. For a given field, we flagged all data points that were more than twice the estimated total flux density of that field. This typically removed about $5-10 \%$ of the data, though for some fields it was as high as $20 \%$.

\subsubsection{Flagging by Statistical Tests}

Removing the "comb" and clipping removed the worst data; however, RFI often shows up as a lower-level effect in many different visibilities that form recognizable patterns that a human can identify, but which are below any reasonable clipping threshold. This RFI usually produces excess signal in one channel for a long time or in many channels for a short time. Statistical tests on the visibility data are necessary to identify this type of RFI. For a given field, we examined the statistical properties for each baseline and polarization separately. The flagging based on these statistical properties represents the final step in our flagging procedure.

For a given field, we examined separately each set of visibilities for a given baseline and polarization. Within this set, each visibility can be identified by its time and frequency, and its amplitude can be represented as a function of these, $S\left(t_{i}, \nu_{j}\right)$, where the time $t_{i}$ and frequency $\nu_{j}$ are those for the $i$ th time interval and $j$ th frequency respectively. There are 120 frequency channels kept after removing attenuated channels at edges of the bandpass and 450 10-second time intervals for a typical observation of 75 minutes duration. This results in a total of 54,000 visibility data points.

First we searched for individual points contaminated by RFI. This was done by calculating the mean and RMS values for the amplitudes of all 54,000 points. We then flagged all points having amplitudes greater than the mean amplitude plus ten times the RMS amplitude.

The second step was to search for RFI-contaminated channels. For this purpose, the time average of all data in each channel, $S_{\nu}\left(\nu_{j}\right)$ was calculated as:

$$
S_{\nu}\left(\nu_{j}\right)=\frac{1}{N_{\text {time }}} \sum_{i=1}^{N_{\text {time }}} S\left(t_{i}, \nu_{j}\right)
$$

where $N_{\text {time }}$ is the number of 10 -second time intervals. We then calculated the median value of $S_{\nu}\left(\nu_{j}\right)$ over all $\nu_{j}$, which we call $S_{\nu, \text { med }}$. We also define $\Delta S_{\nu}$ as the difference between the median and the value of $S_{\nu}\left(\nu_{j}\right)$ for which $25 \%$ of the channels are lower (ie. the difference between the 25 th and 50 th percentiles). For any channel whose median flux exceeded $S_{\nu, \text { med }}$ by more than $6 \Delta S_{\nu}$, we flagged all visibilities in that channel. As RFI can only add power, we only needed to flag channels that are too high, rather than any that might be too low. 
Finally, we searched for times in which all or most channels were contaminated by RFI. This is done in nearly the same way as in step two, but with time and frequency exchanged. For each time, all frequency channels were averaged to give:

$$
S_{t}\left(t_{i}\right)=\frac{1}{N_{\text {chan }}} \sum_{j=1}^{N_{\text {chan }}} S\left(t_{i}, \nu_{j}\right)
$$

where $N_{\text {chan }}=120$ is the number of channels. We then calculated the median value of $S_{t}\left(t_{i}\right)$ over all $t_{i}$, which we call $S_{t, \text { med }}$. We define $\Delta S_{t}$, as the difference between the 25 th and 50 th percentiles of $S_{t}\left(t_{i}\right)$. We flagged all times that had a median flux that exceeded $S_{t, m e d}$ by more than $4 \Delta S_{t}$.

Note from the above discussion that we flagged individual visibilities, channels, and time intervals based on $10 \sigma, 6 \Delta S_{\nu}$ and $4 \Delta S_{t}$ criteria respectively. These levels were determined empirically based on trial and error in order to remove the most RFI possible without removing significant amounts of real features. We erred on the side of leaving in RFI rather than removing real data, and some low-level RFI was inevitably left in much of the data. Again, we emphasize that the best method currently available for flagging RFI is to do so by eye. That being impossible for this survey, our goal was to removed the worst of the RFI, which resulted in acceptable image quality overall.

Figure 2 shows the result of applying our flagging procedure to a baseline with a relatively bad case of RFI contamination. Notice that RFI seemed to affect a set of channels in an almost equally spaced "comb", as described in Section 4.2.2. However, other channels were also contaminated with RFI and were removed. Also seen are horizontal features which are caused by times during which nearly all channels were contaminated. The smooth features, sometimes appearing as diagonal stripes, are from real source structure. As can be seen, most of the worst RFI was successfully removed; however, some low-level contamination remains.

\subsection{Producing Images}

\subsubsection{Channel Averaging}

After flagging, we averaged the data to reduce processing time. The data were averaged down to only 12 channels, the minimum we could retain without introducing significant bandwidth smearing into the images. This resulted in channel widths of $122 \mathrm{kHz}$. Bandwidth smearing occurs in the radial direction with respect to the primary-beam center, and its magnitude is proportional to the distance from the primary-beam center. In our case, a 
point source on the primary beam half-power circle is convolved radially with a function $35.3^{\prime \prime}$ wide. This broadens the radial width of the point-source response from $80^{\prime \prime}$ to $87.4^{\prime \prime}$ at the half-power circle. This effect is of roughly the same magnitude as the smearing from ionospheric effects, which will be discussed later.

\subsubsection{Field-Based Calibration}

Ionospheric phase errors $\phi_{i o n}$ (see Equation 2) must be removed before the $(\mathrm{u}, \mathrm{v})$-data sets are Fourier transformed to make images. At frequencies significantly higher than 74 $\mathrm{MHz}$, the ionospheric phase at any instant is nearly constant across the primary beam of a VLA antenna and can be removed by simple antenna-based calibration. Both ionospheric phases (Kassim et al. 1993) and primary beamwidths scale as $\nu^{-1}$, so the angular size of the "isoplanatic patch" over which the ionospheric phase is constant becomes smaller than the primary beam at low frequencies. At $74 \mathrm{MHz}$ the isoplanatic patch is significantly

smaller than the VLA primary beam, and antenna-based calibration is incapable of removing ionospheric phase errors throughout the field of view. Instead, we must solve for $\phi_{\text {ion }}$ as a function of position within the field of view.

This was done for the survey data with a method called "Field-Based Calibration" (Cotton et al. 2004). Developed specifically for $74 \mathrm{MHz}$ VLA data, this method fits a timevariable phase screen over the field of view. Field-based calibration relies on two main assumptions. First it assumes that the phase screen is the same for all antennas. This is reasonable to assume for the VLA B-configuration because its maximum baseline is $11 \mathrm{~km}$, which is small compared to the size of the isoplanatic patch at the altitude (about $400 \mathrm{~km}$ ) of the maximum electron density of the ionosphere. This calibration method fails for arrays that are much larger than the isoplanatic patch projected onto the ionosphere. The second assumption is that the spatial structure of the ionosphere is smooth enough that across any individual source it can be approximated as a simple linear gradient, which affects source images only by shifting their apparent sky positions. This is true most of the time, but for periods of unusual ionospheric activity this assumption no longer holds, and imaging during these times is not possible with this calibration method.

Field-based calibration is implemented by dividing the data into time intervals short enough that the ionosphere does not vary significantly, generally 1-2 minutes. Within each time interval, small images are produced of sources known to have high flux density after extrapolating from the NVSS with $\alpha=-0.7$. Due to the short time interval these maps have high noise levels (about $1 \mathrm{Jy} /$ beam), and typically only 5-10 sources in a given field will have high enough peak brightness to be clearly detected. These sources can be used 
as ionospheric phase calibrators. We compared their apparent positions at $74 \mathrm{MHz}$ with their NVSS positions and used the offsets to determine the phase gradients at the source positions. A 2nd-order Zernike polynomial phase screen is then fitted to these phase-gradient measurements. Higher-order fits are not possible because there are typically not enough detectable calibrator sources in a field to constrain higher-order terms. However, under normal ionospheric conditions, the 2 nd order Zernike polynomial fit is sufficient to remove most of the ionospheric distortions. Residual ionospheric distortions will be discussed further in later sections on the analysis of image quality.

The improvement in image quality of field-based calibration compared to self-calibration

can be seen in the source maps of Figure 3, which was taken from Cohen et al. (2003). For each map, the dots represent all sources in the image with apparent peak brightnesses of $400 \mathrm{mJy} /$ beam or higher. This $74 \mathrm{MHz}$ VLA data set was observed in the A-configuration, for which ionospheric effects are much more pronounced than in the smaller B- and BnAconfigurations used for the VLSS, and therefore shows a very marked contrast between the resulting image quality of the two methods. The flux density in this field of view is dominated by $3 \mathrm{C}$ 63, which is circled, causing self-calibration to solve for ionospheric phase at that location and subtract this phase over the entire field of view. At increasing angular distances from 3C 63, the ionospheric phases have less correlation with these solutions, and sources appear to have position shifts that vary in time. As the image is produced by averaging the data over time, this causes source smearing which reduces the apparent peak brightness and thus the apparent source density. This is clearly seen in the source map for the self-calibrated image, which shows a declining source density with increasing angular distance from $3 \mathrm{C}$ 63. In contrast, the field-based image has a roughly uniform source density throughout which only decreases at the edges because of primary-beam attenuation.

\subsubsection{Wide-Field Imaging}

After determining the ionospheric phases, the next step is Fourier inversion into the image plane. Normally, this is done with a two-dimensional Fourier inversion of the visibilities by projecting the baseline vectors onto the $u v$-plane perpendicular to the line of sight. However, this is an approximation that is only valid for small fields of view in which $\theta^{2} w_{\text {max }} \ll 1$, where $w_{\max }$ is the maximum baseline component parallel to the line of sight for all visibilities in the data set measured in wavelengths, and $\theta$ is the angular size of the imaged region in radians. The $74 \mathrm{MHz}$ field of view is far too large for this approximation to be valid. Therefore, conversion to the image plane was done with polyhedral imaging that divides the field of view into smaller plane images (facets) inside of which the two-dimensional approximation 
is valid (Cornwell \& Perley 1992; Perley 1999). The facet size was set automatically for each data set such that $\theta^{2} w_{\max }=0.01$, resulting in facets that were typically about $1^{\circ}$ in size or less. Depending on the facet size, each field was covered by between roughly 250 and 1500 facets. Additional facets were placed at the known locations of very strong sources outside of the field of view so that their deconvolution could reduce the effects of their sidelobes within the field of view.

The ionospheric phase screens, determined in Section 4.3.2, were then applied by using the appropriate $\phi_{i o n}$. for each facet as determined by the location of that facet within the phase screen. The facets were deconvolved using the CLEAN algorithm with a constant circular restoring beam with 80" FWHM. The facets were then combined into a single calibrated and astrometrically corrected image of the entire primary beam region.

\subsubsection{Removal of Strong Outlier Sources}

In some cases the location of a field near an unusually strong source could cause high sidelobes in the field of view, which greatly increased the overall noise level. In bad cases it could even cause the ionospheric calibration to fail altogether. In these cases we first self-calibrated the data to the problematic source, then mapped that source and subtracted it from the $u v$-data, and then reversed the calibration before finally proceeding with the imaging as normal. This procedure is generally known as "peeling". This greatly improved the image quality for many fields, although for extremely strong sources, the surrounding fields still have higher noise levels than average because the source subtraction is not perfect and leaves residual errors.

\subsubsection{Corrections of Residual Ionospheric Calibration Errors}

After examining many field images, we discovered that some fraction of them contained residual ionospheric errors that shifted sources from their NVSS positions. There are three potential reasons for VLSS source positions to be different from their NVSS positions: (1) source fitting errors caused by map noise, (2) source centroids being truly different at 74 and $1400 \mathrm{MHz}$ because of spectral variations across the source and (3) the VLSS image at the source location is shifted due to ionospheric calibration errors. Reasons (1) and (2) will cause (usually small) random shifts which are not correlated with the shifts of nearby sources. These shifts are expected and do not require correction. However reason (3) can cause all the sources in a given region to be shifted by nearly the same magnitude and direction, thereby 
making the image astrometrically incorrect in that region. The fact that this was seen in some images (Figure 4, top plot) indicates that ionospheric errors were significantly affecting some of the data.

Ionospheric calibration errors can be separated into errors that are time-dependent and those that are time-independent during the observation. Time-dependent errors could be caused by ionospheric variations that are too complex in time or space to be modeled by our field-based calibration scheme. There is nothing that can be done about these errors because they must be corrected individually for each solution time interval. Each such time interval, by itself, does not produce an image deep enough to detect enough sources to apply a more sophisticated ionospheric model than was originally used.

The main causes of time-independent errors are that one or more of the ionospheric calibrators used has a significantly different centroid at $74 \mathrm{MHz}$ than it does at $1.4 \mathrm{GHz}$ and that the available calibrators are distributed in the field of view in a way that doesn't allow for a realistic solution everywhere in the field. These problems do not vary with time and cause the same calibration error for each time interval. Unlike time-dependent errors, time-independent errors can be further investigated because one can use the data from all time intervals combined, in which generally at least 100 sources are detected in the field of view.

Therefore we implemented an image correction algorithm designed to remove these timeindependent ionospheric calibration errors. We compared the positions of sources in the final $74 \mathrm{MHz}$ image with NVSS positions and fit a up to a 4th-order Zernike polynomial correction, rather than the 2 nd-order correction used for the initial phase calibration. The higher-order correction is possible because of the larger number of available calibrators in the integrated image, rather than the 2-minute snapshots. The large number of available calibrators also allows the algorithm to remove individual sources that give a bad fit to the correction models, making the corrections much more robust against sources with true differences between centroid positions at the two frequencies. The newly modeled "phase screen" was applied in the image plane by stretching and then re-gridding the image. Although not all fields had significant calibration errors, to be thorough and consistent, we applied this correction algorithm to all fields.

The resulting maps show a nearly complete correction of correlated position offsets between VLSS and NVSS as can be seen by comparing the before and after images of Figure 4. We defined the position error of a field as the 85th percentile in distribution of VLSSNVSS source offsets in the field. The 85th percentile was chosen to catch ionospheric errors affecting a small portion of a field without being sensitive to a single source having a large centroid shift between 74 and $1400 \mathrm{MHz}$. For each field, we performed three fits using a 2nd, 
3rd and 4th-order polynomial correction. The fit that produced the lowest position errors (usually, but not always the 4th-order fit) was used for the "final" image. Figure 5 shows the overall improvement in the position errors of all fields. Before correction, $10 \%$ of the fields had position errors greater than 30", with some over $60^{\prime \prime}$. After correction, no field had a position error greater than $30^{\prime \prime}$ and about $95 \%$ had position errors less than $20^{\prime \prime}$, or $1 / 4$ of the beamwidth. Thus the entire survey is accurate to within $30^{\prime \prime}$. Figure 5 also shows that almost no fields have position errors less than $10^{\prime \prime}$. Position uncertainties caused by map noise probably account for this $10^{\prime \prime}$ minimum.

\subsubsection{Image combining}

Individual field maps at this point were still not corrected for the attenuation of the primary-beam pattern, and therefore their sensitivity varied; it was highest at the field centers and tapered off with distance proportional to the primary-beam attenuation. The individual field maps were each truncated at a radius of $6.2^{\circ}$, which is slightly larger than the half-power radius. In order to produce images of nearly uniform sensitivity, the individ-

ual fields were combined using the method of Condon et al. (1998) which corrects for the primary-beam attenuation while co-adding data at each point in the final image weighted proportionally to the inverse square of the estimated RMS noise level in each contributing image. The RMS noise levels were estimated by measuring the noise levels at the centers of the fields and assuming that they increased with radius from the center with inverse proportion to the primary-beam attenuation.

An overlapping grid of square images with $204825^{\prime \prime}$ pixels on a side $\approx 14^{\circ}$ was produced to cover the entire survey region. This grid of combined images comprises the principal data product of the survey. This grid of images was used to produce all sub-images and the source catalog.

\section{Survey Assessment}

\subsection{Sky Area Imaged}

We have now observed, reduced, and publicly released the images and catalogs for the region of the sky shown in Figure 6. This region covers a total of 9.35 sr, about $95 \%$ of the intended survey region $\delta>-30^{\circ}$. Observing time for the observations needed to image the remaining area have been allocated. The full survey data will be released after this is completed and combined with the existing data. 


\subsection{Sensitivity Achieved}

For the sky area shown in Figure 6 we achieved a median RMS noise level of 108 $\mathrm{mJy} /$ beam. However, some regions of the sky had significantly higher noise levels. This can be seen in Figure 7 which quantifies how much sky was observed at each RMS noise level. In the lower plot, one can see that while most of the sky area has now been observed at $100 \pm 30 \mathrm{mJy} /$ beam, there is a significant "tail" extending to much higher noise levels. The main causes for this are: (1) regions near very strong sources in which the RMS noise level is dynamic-range limited, (2) regions at the edges of fields with no neighboring field in which the primary beam shape raises the RMS noise level (3) field located in high-sky-temperature regions in the Galactic plane near the Galactic center, and (4) fields that were affected by unusually bad ionospheric conditions or RFI conditions. We can do nothing about causes (1) and (2), but we intend to re-observe fields affected by causes (3) and (4) to reduce this effect in the next data release.

The sensitivity of an image produced with the $74 \mathrm{MHz}$ VLA is generally not thermal noise limited. For a typical VLSS observation of 75 minutes with 26 working antennas, the theoretical RMS noise is $35 \mathrm{mJy} /$ beam for a system temperature $T_{s y s}$ of $1500 \mathrm{~K}$ and aperture efficiency of 0.15 . This value of $T_{s y s}$, which is dominated by the sky temperature $T_{\text {sky }}$, is typical for the sky far from the Galactic plane, but can be up to twice that on the Galactic plane and even higher still near the Galactic center. However, the map noise is dominated not by $T_{\text {sys }}$, but by sidelobe confusion from the thousands of sources inside and outside the primary beam area, an effect that is accentuated by the poor forward gain and commensurate high sidelobe levels of the $74 \mathrm{MHz}$ primary beam. This is why most fields have noise levels that are between two to four times the thermal noise.

While $T_{s k y}$ is not the dominant factor producing map noise, the fact that it varies greatly over the survey region makes it instructive to look for correlations between map noise and sky position. The top graph of Figure 8 shows the map noise for each field as a function of Galactic latitude for all fields except those very close to extremely strong sources such as Cygnus A. This plot indicates little if any dependence of map noise on Galactic latitude except for a slight increase very close to zero latitude. Images in the Galactic plane region are investigated further in the bottom graph of Figure 8 which plots the map noise of all fields within $10^{\circ}$ of the Galactic plane as a function of Galactic longitude. This plot shows a clear increase of at least 50\% in the average map noise toward the Galactic center. This

shows that while $T_{s k y}$ is generally not a determining factor in the noise levels for most of the sky, it is high enough to increase map noise in the small region of the sky on the Galactic plane and near the Galactic center. 


\subsection{Image Quality}

A sample VLSS sub-image is shown in Figure 9, The crosses indicate identified sources that were included in the VLSS catalog which will be described in Section 6 .

With the VLSS resolution of $80^{\prime \prime}$ most radio sources are either unresolved or just slightly resolved. However, given the large sky area and number of sources identified, a sizable number of very large and resolved sources have been found. Figure 10 shows a sample of some of the largest sources found in the survey. Many of these are well-known objects and we have labeled them by their common radio names.

\section{Extracting the Catalog}

\subsection{Source Finding}

In order to produce a catalog of sources we used the same Gaussian-fitting program used by the NVSS catalog (Condon et al. 1998). This algorithm identifies each "island" of high brightness in an image that is above a specified threshold. The threshold we used was that an island had to have a peak brightness of at least 4.5 times the local RMS noise in a square 100 pixels on a side centered on that island. Each island was fit to a model of up to four Gaussian peaks, which was generally sufficient to accurately model most source structures. Each catalog entry is a single Gaussian peak. If an "island" was fitted by multiple Gaussian peaks, they were cataloged as separate sources. Therefore, a catalog "source" is simply a bright region that was fit by a Gaussian, but may actually be just one component of an astronomical source; for example, one lobe of a double source. After fitting, only sources

with peak brightnesses of 5 times the local RMS noise level $(\sigma)$ or greater were kept in the catalog.

The result was a list of Gaussian fits, each described by six parameters:

- $\alpha=$ right ascension (J2000)

- $\delta=$ declination $(\mathrm{J} 2000)$

- $I_{p}=$ peak brightness

- $\theta_{M}=$ major axis FWHM

- $\theta_{m}=$ minor axis FWHM 
- $\phi_{P A}=$ Position Angle (east of north)

These are all free parameters fit to each source, the only constraint being that $\theta_{M}$ and $\theta_{m}$ are required to be larger than $\theta_{b}=80^{\prime \prime}$, the size of the restoring beam.

\subsection{False Detections}

The $5 \sigma$ cutoff for source detection should eliminate virtually all false source detections for the case of Gaussian map noise. However the noise in the VLSS images is not always Gaussian, mainly because of sidelobes from incompletely deconvolved sources. Therefore in the vicinity of sources with very high peak brightness, we apply a stricter criterion for source detection of $6 \sigma$ peak brightness. This "vicinity" was a circular region around each source with $I_{p}>12 \mathrm{Jy} /$ beam with radius, $\theta_{r}$, that varied according to the measured peak brightness, $I_{p}$, as follows:

$$
\theta_{r}=\left(1^{\circ}\right) \sqrt{\frac{I_{p}}{60 \mathrm{Jy} / \text { beam }}}
$$

up to a maximum of $6^{\circ}$. The parameters of this equation were adjusted empirically in order to remove the most false sources without removing significant numbers of real sources. This removed most but not all source detections that upon visual inspection of the maps were clearly sidelobes of very strong sources. In all, 549 sources were removed in this manner. Of these, 263 (or 48\%) had no NVSS counterpart within 60", whereas for all sources, fewer than $1 \%$ have no NVSS counterpart within $60^{\prime \prime}$. While we cannot use NVSS counterparts to determine if individual sources are real, they are useful in comparing the reliability of large sets of sources. Based on this comparison it is clear that these 549 "sources" were not reliable enough to keep in the survey. However, 286 of these sources did have counterparts, and assuming most of these are real it is clear that real sources were removed from the catalog. Thus we increased the reliability while decreasing the completeness of the catalog in the vicinity of sources with very high peak brightnesses.

\subsection{Derived Parameters}

The six parameters from each Gaussian fit were used along with knowledge of the restoring beam size, always $\theta_{b}=80^{\prime \prime}$, and the local RMS map noise, $\sigma$, to produce the derived parameters for each source that we present in the VLSS catalog. Three of the 
derived parameters are the same as the fitted parameters: $\alpha, \delta$ and $\phi_{P A}$. However, instead of reporting the peak brightness, $I_{p}$, we instead give the integrated flux density $S_{i}$. Also, instead of reporting the fitted source sizes, $\theta_{M}$ and $\theta_{m}$, we use our knowledge of the restoring beam size to calculate deconvolved source sizes, $\phi_{M}$ and $\phi_{m}$. In Section 7 , we describe in detail how these derived parameters and their errors are determined.

\section{Derivation of Source Parameters and Their Accuracy}

\subsection{Source Positions}

The source coordinates, $\alpha$ and $\delta$, are those of the fitted Gaussian. The largest contributors to their RMS errors, $\sigma_{\alpha}$ and $\sigma_{\delta}$, are errors in the ionospheric phase calibration and fitting errors caused by map noise. Thus the total position errors are quadratic sums of two sources of error as are given by the following formulas:

$$
\begin{gathered}
\sigma_{\alpha}^{2}=\sigma_{\alpha, f i t}^{2}+\sigma_{\alpha, c a l}^{2} \\
\sigma_{\delta}^{2}=\sigma_{\delta, f i t}^{2}+\sigma_{\delta, c a l}^{2}
\end{gathered}
$$

where $\sigma_{\alpha, c a l}$ and $\sigma_{\delta, c a l}$ are the position errors due to calibration errors and $\sigma_{\alpha, f i t}$ and $\sigma_{\delta, f i t}$ are the fitting errors.

\subsubsection{Position Errors Caused by Calibration Errors}

Calibration errors affect the positions of all sources, while map noise causes position errors that are inversely proportional to source flux density. Therefore we can isolate the calibration errors by focusing on sources that are strong enough that the fitting errors are negligible in comparison. However, most of the strongest sources were also used as calibrators during field-based calibration. A source used as a calibrator could have a smaller calibrationinduced position error than a typical source. Therefore, we restrict our sample to sources with peak brightness below $3.5 \mathrm{Jy} /$ beam, which is the threshold below which calibrator sources are rejected. This will ensure that the sample remains unbiased.

We can estimate the position error of a source by measuring the difference between its VLSS and NVSS positions. For most sources, the NVSS position errors will be much smaller than the VLSS position errors because: (1) the NVSS resolution is nearly twice that of the VLSS (45" versus 80"), (2) virtually all sources are detected in NVSS at a much higher signal-to-noise ratio than in the VLSS and (3) NVSS calibration errors are known to be only 
about $0.5^{\prime \prime}$ because of smaller ionospheric effects at the higher observing frequency. Thus the difference between the NVSS and VLSS positions can be assumed to be dominated by the VLSS position error. However, there are exceptions to this for sources in which the source centroid is in fact different at $74 \mathrm{MHz}$ than at $1.4 \mathrm{GHz}$ because of a spatially varying spectral index throughout the source (i.e. the core versus a lobe of a radio galaxy). This effect may cause a small contamination when calculating average position offsets for large numbers of sources. However, it can only increase the average offset, and so if anything this method of estimating the position errors will be an overestimate, and therefore a more conservative error estimate.

To examine position errors of strong sources, we used only VLSS sources that have (1) peak brightness less than $3.5 \mathrm{Jy} /$ beam (to avoid calibrator sources), (2) a detection level of at least $30 \sigma,(3)$ a bright $\left(I_{p}>50 \mathrm{mJy} /\right.$ beam) NVSS counterpart within $120^{\prime \prime}$, (4) no other counterpart within $120^{\prime \prime}$, and (5) fitted major axes less than $120^{\prime \prime}$. The fourth and fifth criteria were included to remove fitting errors caused by very large or multiple-component sources having highly non-Gaussian shapes and the increased likelihood of having real source centroid shifts between the two frequencies. There were 866 sources that met these criteria.

We then calculated the VLSS-NVSS position differences for each of these sources, which are plotted in Figure 11. The results yielded average offset of $\Delta \alpha_{\text {mean }}=0.08^{\prime \prime}$ and $\Delta \delta_{\text {mean }}=$ $0.08^{\prime \prime}$ and RMS deviation about mean values of $\Delta \alpha_{R M S}=2.86^{\prime \prime}$ and $\Delta \delta_{R M S}=3.03^{\prime \prime}$. Because we couldn't use the very strongest sources because they might have been used as calibrators, there is some level of contamination in these figures caused by map noise errors. For example, a point source detected at the $30 \sigma$ level (the lowest allowed in the sample) should have a position error in both dimensions of $1.35^{\prime \prime}$, which subtracted in quadrature would reduce $\Delta \alpha_{R M S}$ from $2.86^{\prime \prime}$ to $2.52^{\prime \prime}$ and $\Delta \delta_{R M S}$ from $3.03^{\prime \prime}$ to $2.71^{\prime \prime}$. However, this is only about a $10 \%$ reduction and even this is only for sources at the minimum signal-to-noise ratio in the sample. Therefore, while these average offsets could be slightly overestimated, we will proceed with them as conservative error estimates. Based on these values, we adjusted the VLSS source positions by subtracting the mean offset values and set $\sigma_{\alpha, c a l}=2.86^{\prime \prime}$ and $\sigma_{\delta, c a l}=3.03^{\prime \prime}$ in Equations 6a and 6b.

\subsubsection{Position Errors Caused by Map Noise}

Errors caused by Gaussian fitting in the presence of map noise depend on the signal-

to-noise ratio of the detection, $I_{p} / \sigma$. However, Condon (1997) found that in practice errors 
were more reliably predicted by using the following "effective" signal-to-noise ratio:

$$
\rho^{2}=\frac{\theta_{M} \theta_{m}}{4 \theta_{N}^{2}}\left[1+\left(\frac{\theta_{N}}{\theta_{M}}\right)^{2}\right]^{\alpha_{M}}\left[1+\left(\frac{\theta_{N}}{\theta_{m}}\right)^{2}\right]^{\alpha_{m}} \frac{I_{p}^{2}}{\sigma^{2}}
$$

for which $\theta_{N}$ is the FWHM of the Gaussian noise correlation function which we can take to be the restoring beam size $\theta_{b}$. The exponents $\alpha_{M}$ and $\alpha_{m}$, used for the terms adjusting for the major and minor axes respectively, differ depending on the error parameter being calculated.

The position uncertainties are therefore given by:

$$
\begin{aligned}
\sigma_{M}^{2} & =\frac{\theta_{M}^{2}}{(4 \ln 2) \rho^{2}} \\
\sigma_{m}^{2} & =\frac{\theta_{m}^{2}}{(4 \ln 2) \rho^{2}}
\end{aligned}
$$

where $\sigma_{M}$ and $\sigma_{m}$ are the position errors along the major and minor axes respectively. The exponents used for calculating $\rho$ from Equation 7 are $\alpha_{M}=5 / 2$ and $\alpha_{m}=1 / 2$ for calculating $\sigma_{M}$, and $\alpha_{M}=1 / 2$ and $\alpha_{m}=5 / 2$ for calculating $\sigma_{m}$ (Condon 1997).

To get the source-fitting position errors along the right ascension and declination axes as needed for Equations $6 \mathrm{a}$ and $6 \mathrm{~b}$, we have:

$$
\begin{aligned}
\sigma_{\alpha, f i t}^{2} & =\sigma_{M}^{2} \sin ^{2}\left(\phi_{P A}\right)+\sigma_{m}^{2} \cos ^{2}\left(\phi_{P A}\right) \\
\sigma_{\delta, f i t}^{2} & =\sigma_{M}^{2} \cos ^{2}\left(\phi_{P A}\right)+\sigma_{m}^{2} \sin ^{2}\left(\phi_{P A}\right)
\end{aligned}
$$

These errors are then added in quadrature to the calibration errors that were determined in Section 7.1.1 to produce the total position errors that we include in the VLSS source catalog.

To check the validity of these error estimates, we again compared with the NVSS positions, but we used relatively weak sources so that the source-fitting errors are significant. Sources were selected with the same criteria used in Section 7.1.1, only this time we only selected sources with $5 \sigma<I_{p}<10 \sigma$. There were 18,490 sources that met these criteria, and the resulting position error estimates are shown in Figure 12. All source offsets $(\Delta \alpha, \Delta \delta)$ were divided by the calculated position errors for that source $\left(\sigma_{\alpha}, \sigma_{\delta}\right)$. The ratios of actual source offsets to calculated errors, $\Delta \alpha / \sigma_{\alpha}$ and $\Delta \delta / \sigma_{\delta}$, both had RMS values of 1.12. These values are close to the expected values of unity, and part of the reason they are somewhat higher is likely due to actual source centroid shifts between $74 \mathrm{MHz}$ and $1.4 \mathrm{GHz}$. Therefore we conclude that our error estimates are reasonable. In our catalog, we multiplied our position error estimates by 1.12 . 


\section{2. $\quad$ Source Sizes}

Deconvolved FWHM source sizes, $\phi_{M}$ and $\phi_{m}$, can be calculated as follows:

$$
\begin{aligned}
\phi_{M} & =\sqrt{\left(\theta_{M}^{2}-\theta_{b}^{2}\right)} \\
\phi_{m} & =\sqrt{\left(\theta_{m}^{2}-\theta_{b}^{2}\right)}
\end{aligned}
$$

where $\theta_{M}$ and $\theta_{m}$ are the fitted FWHM source sizes and $\theta_{b}=80^{\prime \prime}$ is the FWHM of the circular restoring beam. Constraining the fitted sizes to be larger than the restoring beam guarantees that the deconvolved source sizes are zero or greater. The position angle, $\phi_{P A}$, is simply the original fitted value. Equations 10a and 10b do not take into account imaging artifacts that increase the fitted size of even a point source beyond that of the restoring beam, such as time-average smearing, bandwidth smearing or, as will be discussed in Section 7.2.1, ionospheric smearing. Therefore, values of $\phi_{M}$ and $\phi_{m}$ are only valid when they are greater than any of these effects are likely to produce. As we estimate in Section 7.2.1 this value is about $45^{\prime \prime}$, which is larger than at least $95 \%$ of ionospheric smearing effects and also larger than any bandwidth or time-average smearing. Below $45^{\prime \prime}, \phi_{M}$ and $\phi_{m}$ should be treated as upper limits on the deconvolved source size.

The errors in the fitted sizes are Gaussian distributed, with RMS error given by:

$$
\begin{aligned}
\sigma^{2}\left(\theta_{M}\right) & =\frac{2 \theta_{M}^{2}}{\rho^{2}}+\epsilon_{o}^{2} \theta_{b}^{2} \\
\sigma^{2}\left(\theta_{m}\right) & =\frac{2 \theta_{m}^{2}}{\rho^{2}}+\epsilon_{o}^{2} \theta_{b}^{2}
\end{aligned}
$$

where the exponents used for calculating $\rho$ from Equation 7 are $\alpha_{M}=5 / 2$ and $\alpha_{m}=1 / 2$ for calculating $\sigma^{2}\left(\theta_{M}\right)$, and $\alpha_{M}=1 / 2$ and $\alpha_{m}=5 / 2$ for calculating $\sigma^{2}\left(\theta_{m}\right)$ (Condon 1997). The term with $\epsilon_{o}$ is a calibration uncertainty which we will estimate in Section 7.2.1,

We consider a source to be significantly resolved if the fitted $\theta_{M}$ is larger than the beam size by more than $2.33 \sigma\left(\theta_{M}\right)$. That is the threshold for which a true point source would appear resolved only $2 \%$ of the time. If an axis is significantly resolved, we estimate the error of the deconvolved value by varying the fitted size by \pm 1 standard deviation and taking half of the difference of the resulting upper and lower deconvolved values. This gives:

$$
\begin{gathered}
\sigma\left(\phi_{M}\right)=\frac{1}{2}\left[\sqrt{\left(\theta_{M}+\sigma\left(\theta_{M}\right)\right)^{2}-\theta_{b}^{2}}-\sqrt{\left(\theta_{M}-\sigma\left(\theta_{M}\right)\right)^{2}-\theta_{b}^{2}}\right] \\
\sigma\left(\phi_{m}\right)=\frac{1}{2}\left[\sqrt{\left(\theta_{m}+\sigma\left(\theta_{m}\right)\right)^{2}-\theta_{b}^{2}}-\sqrt{\left(\theta_{m}-\sigma\left(\theta_{m}\right)\right)^{2}-\theta_{b}^{2}}\right] .
\end{gathered}
$$


If an axis is not significantly resolved, we only report the $98 \%$ confidence upper limits to the deconvolved source size along that axis. If at least one axis is significantly resolved, we give the position angle, $\phi_{P A}$ and its error given by:

$$
\sigma_{P A}^{2}=\frac{4}{\rho^{2}}\left(\frac{\theta_{M} \theta_{m}}{\theta_{M}^{2}-\theta_{m}^{2}}\right)^{2}
$$

where the exponents used for calculating $\rho$ from Equation 7 are $\alpha_{M}=1 / 2$ and $\alpha_{m}=5 / 2$ (Condon 1997).

\subsubsection{Effects of Ionospheric Smearing on Source Sizes}

Ionospheric calibration errors cause artificial smearing of sources and increase their apparent sizes. To examine this effect we considered VLSS sources that are detected at high signal-to-noise ratios because fitting errors on strong sources are much smaller than ionospheric smearing effects typically are.

The test we conducted was to measure the Strehl ratios of the VLSS sources with the highest peak brightnesses. The Strehl ratio is the ratio of the solid angle of the diffraction limited point source response, which in our case is simply the restoring beam (with 80" FWHM throughout the survey) to the actual solid angle of a point source in an image. It is also the amount by which the peak brightness will be degraded in an image. We estimated this by comparing the fitted VLSS source sizes to the expected source sizes based on convolving the NVSS counterpart source size to VLSS resolution. This test measures not only ionospheric smearing, but also time-average smearing and bandwidth smearing. However, for most sources, incorrect ionospheric calibration is the largest potential cause of smearing.

To avoid other errors, we restricted our sample set to VLSS sources with detection levels of at least $60 \sigma$ and with NVSS counterparts that were small enough to imply a VLSS source size of no more than 1.05 times the restoring beam solid angle. This resulted in 975 sources, and the resulting distribution of Strehl ratios is shown in Figure 13. Because of measurement errors a small fraction of sources have a Strehl ratio above 1, which is unphysical. Most sources however had Strehl ratios below 1, with a median value of 0.96. There is a long tail of values well below the median, which could be due to observations with particularly high ionospheric calibration errors. Equally plausible is that some fraction of sources have sizes that really do appear larger at $74 \mathrm{MHz}$ than at $1.4 \mathrm{GHz}$ because of diffuse steep-spectrum emission. This effect has been previously observed in sources such as Hydra 
A (Lane et al. 2004). Finally, one may question whether the use of the sources with the highest peak brightnesses, many of which were used as calibrator sources, would be biased toward higher Strehl ratios. This was checked by taking a similar sample of sources with peak brightness below the $3.5 \mathrm{Jy} /$ beam cutoff for calibrator selection. The weaker source sample actually had a slightly higher median Strehl ratio of 0.97 , but with a much larger spread which is likely caused by the increased effects of map noise on weaker sources. However, it does not seem that the source sample is biased toward higher-than-normal Strehl ratios, and therefore our initial test appears reliable. Based on the median Strehl ratio of 0.96, we use $\epsilon_{o}=0.02$ in Equations $11 \mathrm{a}$ and $11 \mathrm{~b}$ assuming that on average each axis will have half of the residual ionospheric smearing.

This test indicates that ionospheric smearing reduced the peak brightness of the typical source by about 4\%. Flux density is not lost, just spread out, so the integrated flux density should theoretically not be affected. However, the reduction in peak brightness will cause some sources to fall below the $5 \sigma$ detection threshold, which affects the completeness of the source catalog.

The biggest effect of ionospheric smearing is to increase the estimated source sizes. While a Strehl ratio of 0.96 has a rather small effect on the fitted source size, it has a larger effect on the deconvolved source size estimate. For a point source, if we take the worst case scenario, in which the entire source smearing was along one axis, a Strehl ratio of 0.96 would correspond to a deconvolved major axis of $23^{\prime \prime}$. A Strehl ratio of 0.87 (the value of the lowest 95th percentile in the distribution of Figure 13) would increase the deconvolved major axis to $45^{\prime \prime}$.

In the catalog, we report the deconvolved source sizes based only on the source fitting without taking into account the effects of ionospheric smearing. This is for two main reasons. First, though we can quantify this effect statistically for a large number of sources, it is nearly impossible to estimate the effect for a single source. Second, for all sources except those with very high peak brightnesses, any smearing is likely to be small compared to the normal map noise induced fitting errors, and would not significantly effect the reported source sizes. However, the user of the catalog should be warned that source sizes smaller than about $45^{\prime \prime}$ should be treated as upper limits.

\subsection{Flux Densities}

In this section we describe how the source flux densities and their errors were determined. The flux-density error estimates are quadratic sums of map-noise-induced fitting 
errors (Section 7.3.1) and intensity-proportional flux errors produced by errors in both the flux-density scale and primary-beam corrections (Sections 7.3.2 and 7.3.3). We also correct the flux densities for a clean bias which we discuss in Section 7.3.4. In Section 7.3.5, we compare our flux values to those from other notable low-frequency surveys as a general check of our results.

\subsubsection{Flux-Density Errors from Map Noise}

The map noise error depends on the effective signal to noise ratio, $\rho$, which for flux densities should be calculated using Equation 7 with $\alpha_{M}=\alpha_{m}=3 / 2$ (Condon 1997). Also, map noise error depends on the number of degrees of freedom allowed in the Gaussian fit. Therefore, the best estimate for the integrated flux density of a source depends on whether or not the source is significantly resolved (Condon 1997). There are three cases to consider:

1. If both the major and minor axes are significantly resolved, the integrated flux density is

$$
S_{i}=I_{p}\left(\frac{\theta_{M} \theta_{m}}{\theta_{b}^{2}}\right)
$$

and the RMS uncertainty in $S_{i}$ is

$$
\sigma\left(S_{i}\right)=S_{i}\left(\epsilon_{S}^{2}+\frac{2 I_{p}^{2}}{\rho^{2}}+\frac{\theta_{b}^{2}}{\theta_{M} \theta_{m}}\left[\frac{\sigma^{2}\left(\theta_{M}\right)}{\theta_{M}^{2}}+\frac{\sigma^{2}\left(\theta_{m}\right)}{\theta_{m}^{2}}\right]\right)^{1 / 2}
$$

2. If the major axis is significantly resolved but the minor axis is not, the best estimate for the integrated flux density is

$$
S_{i}=I_{p}\left(\frac{\theta_{M}}{\theta_{b}}\right)\left(\frac{\theta_{m}}{\theta_{b}}\right)^{1 / 2}
$$

and the RMS uncertainty in $S_{i}$ is

$$
\sigma\left(S_{i}\right)=S_{i}\left(\epsilon_{S}^{2}+\frac{3 I_{p}^{2}}{2 \rho^{2}}+\frac{\theta_{b}}{\theta_{M}} \frac{\sigma^{2}\left(\theta_{M}\right)}{\theta_{M}^{2}}\right)^{1 / 2}
$$

3. If neither the major nor minor axis is significantly resolved, the best estimate for the integrated flux density is

$$
S_{i}=I_{p}\left(\frac{\theta_{M} \theta_{m}}{\theta_{b}^{2}}\right)^{1 / 2}
$$

and the RMS uncertainty in $S_{i}$ is

$$
\sigma\left(S_{i}\right)=S_{i}\left(\epsilon_{S}^{2}+\frac{I_{p}^{2}}{\rho^{2}}\right)^{1 / 2} .
$$




\subsubsection{Accuracy of the Flux-Density Scale}

The flux-density scale for each VLSS observation was determined by comparing scans on Cygnus A to a pre-existing model that was scaled to the flux density determined by Baars et al. (1977). As the $74 \mathrm{MHz}$ VLA is a fairly new system, the accuracy of this method is not well determined. Ideally we could compare our results for very strong sources to some pre-existing database of accurate $74 \mathrm{MHz}$ flux densities. However, no database of $74 \mathrm{MHz}$ flux densities exists, nor does any database of low-frequency source spectra with enough accuracy for us to measure the accuracy of our own flux-density scale.

Fortunately, Cygnus A was not the only calibrator we observed. At times when Cygnus A was not at high enough elevation, two other calibrators, Virgo A and 3C 123, were observed in case the calibration from other times on Cygnus A was not sufficient. This never was the case, so these other calibrators were never actually used in data reduction. However, as they are sources observed on many different days, their derived flux densities on each day can be used as an internal check of the flux calibration consistency. 3C 123 was observed on 24 separate days, and the flux-density measurements give a mean value of $390.5 \mathrm{Jy}$ with an RMS scatter of $15.7 \mathrm{Jy}$, indicating that the flux-density scale is consistent to within $4 \%$. This

measurement agrees well with the value predicted by Kühr et al. (1981) which is 387.6 Jy indicating that flux-density scale remains linear even at Cygnus A flux density levels. Virgo A was observed on 42 days, yielding a mean flux density of 1911.5 Jy with an RMS scatter of 119.2 Jy which indicates that the flux-density scale is consistent to within $6 \%$. This is somewhat different than the predicted Kühr et al. (1981) value of $2281.4 \mathrm{Jy}$. However, the Kühr et al. (1981) spectrum is even farther off from its own $80 \mathrm{MHz}$ data point of $1519 \mathrm{Jy}$, indicating that their spectrum may not be a good fit to the data. The fact that Virgo A is a large, heavily resolved source may also be a factor. To be on the conservative side, and also because Virgo A was observed more often, we consider the $6 \%$ to be a reasonable estimate of the accuracy of our flux-density scale.

\subsubsection{Total Intensity-Proportional Flux-Density Errors}

The errors in the flux-density scale, discussed in Section 7.3.2, are only part of the total intensity-proportional flux errors. The other significant component is the primarybeam correction error. The primary beam of a VLA antenna at $74 \mathrm{MHz}$ is not perfectly understood and is probably not symmetric. The analysis of Section 7.3 .2 only considered sources at the center of the field of view, for which primary-beam corrections were not necessary. In this section we analyze the contribution of primary-beam correction errors to the intensity-proportional flux error of any VLSS sources. 
To determine the total intensity-proportional flux errors for a general VLSS source, we take advantage of the fact that most sources have been observed in two or three different pointings because of the overlapping nature of the observational grid. Thus the original field maps, prior to their combination with adjacent fields in the mosaic, contain multiple flux-density measurements with independent flux-density calibrations and primary-beam corrections. For sources detected at sufficiently high signal-to-noise ratios, map noise errors are negligible compared with intensity-proportional flux errors. Therefore, for strong sources, the differences among flux-density measurements made in overlapping fields are due almost entirely to intensity-proportional flux errors.

We considered all sources detected with signal-to-noise ratios of at least 60 and that had fitted Gaussian major axes less than 120" (1.5 times the restoring beam) to remove sources that are large enough that they may not be well fit by a Gaussian. There were 1,126 such sources and 1,787 pairs of flux-density measurements of the same source from different fields. The root-mean-square of the fractional differences in these measurement pairs indicates a total intensity-proportional flux error of $12.1 \%$. This includes both the flux-density scale errors and primary-beam correction errors. Taking the estimated flux-density scale error of $6 \%$ from Section 7.3 .2 , the primary-beam correction error alone is about $10.5 \%$.

The $12.1 \%$ value for the intensity-proportional flux error is almost certainly an overestimate for two reasons. First, because we only consider sources observed in two or more adjacent fields, we are biased toward sources near the outer region of the primary beam, for which primary-beam corrections will have greater than average errors. Second, this is the estimated error for a single observation. Most VLSS sources are a weighted sum of adjacent field images, and so the averaged error will be somewhat less than the error in a single field. In fact, the comparisons to other catalogs presented later in Section 7.3.5 do indicate that the intensity-proportional flux errors are probably somewhat smaller than this. In particular, the comparisons to $6 \mathrm{C}$ and $8 \mathrm{C}$ sources shows that our intensity-proportional error can be no higher than $10.4 \%$ and even this includes the errors from both of those surveys as well as errors in interpolation which assume a constant spectral index. Therefore, we use $10 \%$ as a conservative estimate of the intensity proportional error and set $\epsilon_{S}=0.10$ for the flux-density error determinations of Equations [15, 17 and 19.

It should be noted that $\epsilon_{S}=0.10$ is the measure of the consistency of our flux-scale and primary-beam corrections with respect to the Baars et al. (1977) scale. That scale itself has an absolute accuracy that they estimate to be $5 \%$ at frequencies below about $300 \mathrm{MHz}$. 


\subsubsection{Clean Bias}

Images deconvolved using the CLEAN algorithm are known to suffer from a "clean bias". This occurs because, as cleaning proceeds to deeper levels, the probability increases that a sidelobe of a source or a noise fluctuation (or a combination of these) can produce a peak higher than any remaining flux in the image. Cleaning this false source results in flux being subtracted from the true source that produced that sidelobe. Therefore the clean bias results in the flux densities of sources being systematically reduced. The magnitude of the bias is independent of the flux density of sources, but is known to increase with higher map noise.

We have conducted a simulation to determine the clean bias in our images. This was done by adding artificial point sources to the $u v$-data and comparing the input flux densities to the measured flux densities in the resulting maps. This is a standard way to measure the clean bias, however the fact that we conducted field-based ionospheric calibration during imaging complicated this simulation. Because ionospheric phase corrections are applied to the data during imaging, we had to apply the inverse of these corrections to the artificial sources before adding them to the $u v$-data in order to produce the intended artificial point sources in the final maps.

In all, we produced 2362 artificial sources at random positions in fields chosen from the survey data to represent a wide range of right ascension and declination. The flux densities varied randomly from 1 to $10 \mathrm{Jy}$. The final results showed a systematic bias between the input flux densities and the map results, confirming the clean bias. The measured clean bias was independent of the input flux density, but did increase with local RMS maps noise, $\sigma$. Fitting a model of the clean bias where its value is proportional to $\sigma$, we found the average clean bias reduces the flux densities of point sources by $1.39 \sigma$. We have corrected the source catalog for this bias by adding this amount, to the peak brightness, $I_{p}$, of each source when calculating the flux density, $S_{i}$, as described in Section 7.3.1. Though larger than the local RMS map noise, the clean bias is still smaller than the flux density error for any source because of the intensity-proportional errors. Therefore, even with the clean bias correction, flux density errors are still dominated by the map noise errors and intensity-proportional errors calculated previously.

The magnitude of the clean bias we find is similar to that of other surveys. It is a bit higher than the value found in the NVSS survey, which is about 0.67 times the typical map noise (Condon et al. 1998). It is a bit less than the value found in the FIRST survey, which is about 1.67 times the typical map noise (Becker et al. 1995). We note that both the NVSS and FIRST use a constant clean bias correction, rather than one that is proportional to the local RMS noise as we do, and the ratios mentioned are relative to the average noise levels 
of those surveys.

\subsubsection{Comparisons with other low-frequency data}

Though it proved impossible to use other low-frequency catalogs to predict $74 \mathrm{MHz}$ flux densities of enough sources with enough precision to test the accuracy of our own fluxdensity scale, comparisons to other low-frequency data are still of interest. In this section, we describe a few of the comparisons we conducted.

Kühr et al. (1981) Spectra: We have compared the flux densities of strong VLSS sources to their expected values based on fitted spectra provided in Kühr et al. (1981), which also uses the flux-density scale of Baars et al. (1977). We used sources with VLSS integrated flux densities between $15 \mathrm{Jy} \leq S_{74} \leq 200 \mathrm{Jy}$ that also had Kühr et al. (1981) spectra based on data including at least one measurement below $100 \mathrm{MHz}$. There were 94 such sources, and the ratio of VLSS flux densities to the Kühr et al. (1981) predicted flux densities had a RMS scatter of $\pm 20 \%$ and a mean value of $0.87 \pm 0.02$.

This ratio seems rather low, and the scatter quite high. One problem with this method is that the errors in most Kühr et al. (1981) spectra tend to be quite large at the lowfrequency end. In particular, the fitted spectra were often quite far off the actual measurement values below $100 \mathrm{MHz}$. In fact, for sources with $80 \mathrm{MHz}$ measurements, the difference between the Kühr et al. (1981) fitted spectrum and the $80 \mathrm{MHz}$ value was often greater than the difference between the $74 \mathrm{MHz}$ VLSS value and the fitted spectrum.

$80 \mathrm{MHz}$ data: Because of the problems experienced in comparing VLSS flux densities to the Kühr et al. (1981) spectra, we chose to simply compare the VLSS measurements directly to the $80 \mathrm{MHz}$ measurements. The $80 \mathrm{MHz}$ measurements were taken with the Culgoora radioheliograph (Slee \& Higgins 1973, 1975; Slee 1977) and adjusted to the Baars et al. (1977) flux-density scale by Kühr et al. (1981). We adjusted these $80 \mathrm{MHz}$ values to predicted $74 \mathrm{MHz}$ values by assuming a spectral index of $\alpha=-0.8$. For the 70 sources with $80 \mathrm{MHz}$ measurements in the Kühr et al. (1981) spectra, the ratio of VLSS to the resulting predicted flux densities had an RMS scatter of $\pm 12 \%$ and a mean value of $0.93 \pm 0.01$, which is a better ratio and scatter than the comparisons to the Kühr et al. (1981) spectra. Because this value depends on the assumed average spectral index, it should be considered as a lower limit, as a significant fraction of sources could have flattened due to synchrotron self-absorption and would have spectra flatter than the typical value of $\alpha=-0.8$. If so, this would raise mean flux-density 
ratio. For example, if we assume an average spectral index of $\alpha=-0.4$, the derived mean flux-density ratio becomes $0.96 \pm 0.01$.

8C to $6 \mathrm{C}$ interpolation: Another comparison to external data was done by predicting the flux at $74 \mathrm{MHz}$ by interpolating between values from the $38 \mathrm{MHz} 8 \mathrm{C}$ catalog (Rees 1990) and the $151 \mathrm{MHz} 6 \mathrm{C}$ catalog (Hales, Baldwin, \& Warner 1988). Choosing VLSS sources that were detected above at least the $60 \sigma$ level and also had single counterparts within $120^{\prime \prime}$ in both the $8 \mathrm{C}$ and $6 \mathrm{C}$ catalogs resulted in a sample of 201 sources. The 8C and 6C catalogs are based on the flux-density scale of Roger et al. (1973), and so we adjusted the interpolated flux-density value to the Baars et al. (1977) scale according to the flux ratios of Cygnus A. Figure 14 shows a comparison of the flux densities of the VLSS sources versus the interpolated predictions. For these sources the average ratio of VLSS to expected flux density had an RMS scatter of $\pm 10.4 \%$ and a mean value of $0.99 \pm 0.01$. This test had a final ratio quite close to unity as well as a low scatter. After taking into account the errors in the $8 \mathrm{C}$ and $6 \mathrm{C}$ catalogs, this result seems consistent with our flux-density scale error estimate of $6 \%$, and actually lower than our admittedly conservative estimate of the total intensity-proportional flux-density error.

\section{Completeness of the Catalog}

In this section we discuss the point-source completeness of the VLSS catalog. Because the RMS noise levels are not constant throughout the survey region, we estimated not the total completeness, but the differential completeness as a function of signal-to-noise ratio (the ratio of the peak brightness of a point source to the local RMS noise level). This was estimated through simulations in which artificial sources were added at random positions to the actual VLSS images. The same source-finding methods were then applied to the maps to see what fraction of artificial sources were "detected".

Simulations were first done for point sources without taking into account any ionospheric source smearing. Gaussians were added to the VLSS images with the same dimensions as the restoring beam (circular with FWHM of $80^{\prime \prime}$ ). The peak brightnesses of the artificial

sources were set to various multiples of the local RMS noise levels and reduced by the clean bias of $1.39 \sigma$ as determined in Section 7.3.4. For a given signal-to-noise ratio, 32 artificial sources were added at random positions to each of the $27914^{\circ} \times 14^{\circ}$ VLSS images that had no blanked regions, for a total sample of 8,928 artificial sources. The fraction of these that were "detected" according to our source-finding criteria was taken to be the differential completeness level at that signal-to-noise ratio. These values are plotted in Figure 15 as the filled points. Because the source finding criteria used included the $5 \sigma$ fitted peak brightness, 
it does not apply to the regions surrounding high peak brightness sources for which a $6 \sigma$ selection was used as described in Section 6.2

We performed a second set of simulations which aimed to factor in residual ionospheric smearing. The Strehl ratio distribution of strongly detected, compact VLSS sources found in Section 7.2.1 was used as a guide. If one assumes that the majority of the smearing occurs on one axis, that Strehl ratio roughly corresponds to a mean deconvolved major axis of $22.9^{\prime \prime}$ with an RMS deviation of $14.2^{\prime \prime}$. Therefore we repeated the same simulation described above, but this time convolving each artificial source with a Gaussian with major axis equal to a Gaussian random variable with a mean of $22.9^{\prime \prime}$ and RMS of $14.2^{\prime \prime}$, a minor axis equal to zero and a PA set randomly between $0^{\circ}$ and $360^{\circ}$. (If the random major axis was less than zero, it was replaced with zero.) The peak brightness of each artificial source was thus lowered by the same factor that its area was increased due to smearing. The resulting differential completeness levels are also shown in Figure 15 as the open points. This simulation indicates that ionospheric smearing has most likely reduced the completeness of the survey to some degree, with the effect being greatest for the faintest sources. The completeness level for $6 \sigma$ point sources is lowered by $16 \%$, however the difference falls to $12 \%$ for $7 \sigma$ sources and

$6 \%$ for $8 \sigma$ sources. A much larger effect on completeness is caused by the clean bias, which shifts the entire differential completeness curve of Figure 15 to the right by 1.39 in units of the local RMS noise level. Taking into account both ionospheric smearing and the clean bias, the $50 \%$ point-source detection limit of the survey is roughly $7 \sigma$, or about 0.7 Jy for a typical noise level of $0.1 \mathrm{Jy} /$ beam.

\section{Accessing the Data}

As the VLSS was conducted as a service to the astronomical community, we have publicly released all images and catalogs as soon as they were reduced and verified. Along with an overall description of the VLSS project and its current status, all verified data can be obtained at the VLSS website:

http://lwa.nrl.navy.mil/VLSS

The data are available in several forms. First, all images in the grid of the original large $\approx 14^{\circ} \times 14^{\circ}$ combined images are available to be directly downloaded as FITS files. Further, we have made available an online postage-stamp server in which a smaller image around a point of interest can be obtained for any user-entered coordinates within the currently completed survey region. 
The current VLSS catalog file can be downloaded as well. Also provided is browser software which uses the catalog file to search for sources within a given radius of given positions using various criteria and gives the final parameters and their error estimates. A small sample the output of the VLSS catalog browser is shown in Table 3 .

\section{Conclusion and Future Work}

We have nearly completed a $74 \mathrm{MHz}$ survey of the entire sky visible from the northern hemisphere. This was done by overcoming the large ionospheric effects which have been the main obstacle to wide-field arcminute-resolution imaging at this frequency for the last 50 years. We now have high-quality $74 \mathrm{MHz}$ images for roughly $3 \pi$ sr. of sky, yielding nearly 70,000 sources detected at the $5 \sigma$ level or greater in the images. All VLSS data are available publicly at the VLSS website:

\section{http://lwa.nrl.navy.mil/VLSS}

In terms of both sky coverage and resolution, we now have produced a low frequency equivalent to the most comprehensive radio sky survey yet produced, the NVSS. The one aspect that keeps the VLSS from being a full equivalent to the NVSS is sensitivity. The VLSS has a typical RMS noise level roughly 220 times higher than the NVSS, and even allowing for an average spectral index of $\alpha=-0.8$, it is still less sensitive than the NVSS by a factor of 20. As discussed in Section 5.2, the limiting factor in VLSS sensitivity is sidelobe confusion. A telescope designed specifically for low frequencies could overcome this problem to a large extent with better forward gain, a smaller field of view, and higher resolution. Yet, even without sidelobe confusion, thermal noise still prevents low-frequency observations from attaining sensitivity anywhere near that of cm-wavelengths. This is because of the very high sky noise temperature at low frequencies caused by Galactic synchrotron emission. The only solution to this is a telescope with vastly more collecting area than the VLA. A number of low frequency instruments are being planned (ie. LWA, LOFAR) which will have huge collecting areas. It is these instruments which will truly bring low-frequency observations up to the level of cm-wavelength capabilities. It is hoped that the VLSS project will provide both inspiration (in the form of observational experience and science) and information (in the form of a sky model and ionospheric data) for the design and operation of these new instruments. 


\section{Acknowledgments}

Basic research in radio astronomy at the Naval Research Laboratory is supported by the office of Naval Research. The National Radio Astronomy Observatory is a facility of The National Science Foundation operated under cooperative agreement by Associated Universities, Inc. We thank Joseph Helmboldt for helping to diagnose errors in the source flux-density measurements.

\section{REFERENCES}

Baars, J. W. M., Genzel, R., Pauliny-Toth, I. I. K., \& Witzel, A. 1977, A\&A, 61, 99

Becker, R. H., White, R. L., \& Helfand, D. J. 1995, ApJ, 450, 559

Bock, D. C.-J., Large, M. I., \& Sadler, E. M. 1999, AJ, 117, 1578

De Breuck, C., van Breugel, W., Röttgering, H. J. A., \& Miley, G. 2000, A\&AS, 143, 303

Chambers, K. C., Miley, G. K., \& van Breugel, W. 1987, Nature, 329, 604

Cohen, A. S., et al. 2003, ApJ, 591, 640

Condon, J. J. 1997, PASP, 109, 166

Condon, J. J., Cotton, W. D., Greisen, E. W., Yin, Q. F., Perley, R. A., Taylor, G. B., \& Broderick, J. J. 1998, AJ, 115, 1693

Cornwell, T. J., \& Perley, R. A. 1992, A\&A, 261, 353

Cotton, W. D., Condon, J. J., Perley, R. A., Kassim, N., Lazio, J., Cohen, A., Lane, W., \& Erickson, W. C. 2004, Proc. SPIE, 5489, 180

Enßlin, T. A., Röttgering, H. 2002, A\&A, 396, 83

Hales, S. E. G., Baldwin, J. E., \& Warner, P. J. 1988, MNRAS, 234, 919

Kassim, N. E., Perley, R. A., Erickson, W. C., \& Dwarakanath, K. S. 1993, AJ, 106, 2218

Kassim, N. E., Perley, R. A., Dwarakanath, K. S., \& Erickson, W. C. 1995, ApJ, 455, L59

Kassim, N. E., Lazio, T. J. W., Erickson, W. C., Perley, R. A., Cotton, W. D., Greisen, E. W., Cohen, A. S., Hicks, B. \& Schmitt, H. R. 2007, accepted for publication in ApJS, arXiv:0704.3088v1 on astro-ph 
Kühr, H., Witzel, A., Pauliny-Toth, I. I. K., \& Nauber, U. 1981, A\&AS, 45, 367

Lane, W. M., Clarke, T. E., Taylor, G. B., Perley, R. A., \& Kassim, N. E. 2004, AJ, 127, 48

Lane, W. M., Cohen, A. S., Kassim, N. E., Lazio, T. J. W., Perley, R. A., Cotton, W. D., \& Greisen, E. W. 2005, Radio Science, 40, 5

Perley, R. A. 1999, ASP Conf. Ser. 180: Synthesis Imaging in Radio Astronomy II, 180, 383

Rees, N. 1990, MNRAS, 244, 233

Rengelink, R. B., Tang, Y., de Bruyn, A. G., Miley, G. K., Bremer, M. N., Roettgering, H. J. A., \& Bremer, M. A. R. 1997, A\&AS, 124, 259

Roger, R. S., Costain, C. H., \& Bridle, A. H. 1973, AJ, 78, 1030

Slee, O. B., \& Higgins, C. S. 1973, Australian Journal of Physics Astrophysical Supplement, 27,1

Slee, O. B., \& Higgins, C. S. 1975, Australian Journal of Physics Astrophysical Supplement, 36,1

Slee, O. B. 1977, Australian Journal of Physics Astrophysical Supplement, 43, 1

Slee, O. B., Roy, A. L., Murgia, M., Andernach, H., \& Ehle, M. 2001, AJ, 122, 1172

Wall, J. V., \& Jackson, C. A. 1997, MNRAS, 290, L17

White, R. L., Becker, R. H., Helfand, D. J., \& Gregg, M. D. 1997, ApJ, 475, 479 
Table 1. VLSS Project Parameters

\begin{tabular}{ll}
\hline \hline \multicolumn{1}{c}{ Parameter } & \multicolumn{1}{c}{ Value } \\
\hline Frequency & $73.8 \mathrm{MHz}$ \\
Total Bandwidth & $1.56 \mathrm{MHz}$ \\
Channel Width & $12.2 \mathrm{kHz}$ \\
Channel Width (averaged) & $122 \mathrm{kHz}$ \\
Resolution & $80^{\prime \prime}$ \\
RMS noise level & $100 \mathrm{mJy} /$ beam (typical) \\
Survey Region & all sky above $\delta>-30^{\circ}$ \\
Survey Area & $3 \pi$ sr. \\
Sources Detected $(\geq 5 \sigma)$ & $68,311$ (as of 2007$)$ \\
\hline
\end{tabular}


Table 2. Observation Dates for the VLSS

\begin{tabular}{lccr}
\hline \hline Project & Dates & Configuration & Total Time (hrs) \\
\hline AP 397 & 9,26 Feb 2001 & B & 36 \\
AP 441 & 8-20 Jun 2002 & B & 64 \\
AP 452 & 20 Sep - 6 Oct 2003 & BnA & 69 \\
& 18 Oct - 7 Dec 2003 & B & 283.5 \\
& 24 Jan - 14 Feb 2005 & BnA & 96 \\
& 19 Feb - 7 Apr 2005 & B & 289.5 \\
& 3 Jun - 9 Jun 2006 & BnA & 43.5 \\
AP 509 & Fall 2007 & BnA & 10 \\
& Fall 2007 & B & 25.5 \\
\hline
\end{tabular}


Table 3. Sample Section from the VLSS Source Catalog

\begin{tabular}{|c|c|c|c|c|c|c|c|c|c|c|}
\hline & Source & $\begin{array}{c}\alpha(\mathrm{J} 2000) \\
(\mathrm{h}, \mathrm{m}, \mathrm{s})\end{array}$ & $\begin{array}{c}\delta(\mathrm{J} 2000) \\
\left({ }^{\circ},{ }^{\prime},{ }^{\prime \prime}\right)\end{array}$ & $\underset{\text { Jy }}{\text { Flux }}$ & maj. $\left({ }^{\prime \prime}\right)$ & $\begin{array}{c}\text { Size } \\
\text { min. }\left({ }^{\prime \prime}\right)\end{array}$ & $\mathrm{PA}\left({ }^{\circ}\right)$ & Field & $\mathrm{X}_{p i x}$ & $\mathrm{Y}_{p i x}$ \\
\hline VLSS & J0917.9+5853 & $\begin{array}{rr}09 & 1759.45 \\
& 0.59\end{array}$ & $\begin{array}{r}+585326.3 \\
4.7\end{array}$ & $\begin{array}{l}1.28 \\
0.15\end{array}$ & $<57.1$ & $<56.8$ & & $0900+550$ & 691 & 1596 \\
\hline VLSS & J0917.9+0034 & $\begin{array}{rr}091759.51 \\
0.35\end{array}$ & $\begin{array}{r}+003448.3 \\
5.3\end{array}$ & $\begin{array}{l}1.13 \\
0.14\end{array}$ & $<62.8$ & $<62.6$ & & $0920+050$ & 1097 & 389 \\
\hline VLSS & J0918.0+3001 & $\begin{array}{rr}09 & 1801.15 \\
0.38\end{array}$ & $\begin{array}{r}+300128.5 \\
5.1\end{array}$ & $\begin{array}{l}0.94 \\
0.12\end{array}$ & $<61.2$ & $<60.4$ & & $0936+350$ & 1585 & 322 \\
\hline VLSS & J0918.0-0303 & $\begin{array}{rr}09 & 1801.38 \\
& 0.52\end{array}$ & $\begin{array}{r}-030353.1 \\
7.6\end{array}$ & $\begin{array}{l}0.69 \\
0.11\end{array}$ & $<86.1$ & $<81.8$ & & $0920-050$ & 1096 & 1304 \\
\hline VLSS & J0918.0-1303 & $\begin{array}{rr}09 & 1802.53 \\
0.31\end{array}$ & $\begin{array}{rr}-1303 & 13.8 \\
4.8\end{array}$ & $\begin{array}{l}2.41 \\
0.28\end{array}$ & $<82.6$ & $<53.2$ & & $0920-150$ & 1094 & 1305 \\
\hline VLSS & J0918.0-1205 & $\begin{array}{rr}09 & 1803.63 \\
0.20\end{array}$ & $\begin{array}{rr}-1205 & 02.0 \\
3.0\end{array}$ & $\begin{array}{r}579.59 \\
58.05\end{array}$ & $\begin{array}{r}186.0 \\
1.8\end{array}$ & $\begin{array}{r}78.1 \\
2.3\end{array}$ & $\begin{array}{r}9.1 \\
0.10\end{array}$ & $0920-150$ & 1093 & 1445 \\
\hline VLSS & $\mathrm{J} 0918.0+4311$ & $\begin{array}{rr}09 & 1804.32 \\
0.47\end{array}$ & $\begin{array}{r}+431157.2 \\
5.2\end{array}$ & $\begin{array}{l}1.12 \\
0.14\end{array}$ & $<62.0$ & $<61.8$ & & $0936+450$ & 1495 & 779 \\
\hline VLSS & J0918.0-2402 & $\begin{array}{rr}09 & 1804.98 \\
0.25\end{array}$ & $\begin{array}{r}-240230.8 \\
3.4\end{array}$ & $\begin{array}{l}2.95 \\
0.31\end{array}$ & $<50.4$ & $<38.6$ & & $0900-250$ & 431 & 1153 \\
\hline VLSS & J0918.1+6537 & $\begin{array}{rr}09 & 1807.02 \\
0.67\end{array}$ & $\begin{array}{r}+653716.8 \\
4.3\end{array}$ & $\begin{array}{l}1.39 \\
0.16\end{array}$ & $<51.5$ & $<51.5$ & & $0920+650$ & 1053 & 1115 \\
\hline VLSS & J0918.1+3225 & $\begin{array}{rr}09 & 1807.17 \\
0.35\end{array}$ & $\begin{array}{r}+322528.1 \\
4.5\end{array}$ & $\begin{array}{l}1.49 \\
0.17\end{array}$ & $<79.2$ & $<50.2$ & & $0936+350$ & 1568 & 666 \\
\hline VLSS & J0918.1+1227 & $\begin{array}{rr}09 & 1809.85 \\
0.20\end{array}$ & $\begin{array}{rr}+122746.1 \\
& 3.1\end{array}$ & $\begin{array}{l}6.08 \\
0.64\end{array}$ & $\begin{array}{r}31.8 \\
6.4\end{array}$ & $<38.2$ & $\begin{array}{r}84.7 \\
24.34\end{array}$ & $0920+150$ & 1090 & 660 \\
\hline VLSS & J0918.1-0841 & $\begin{array}{rr}09 & 18 \quad 10.84 \\
& 1.06\end{array}$ & $\begin{array}{r}-084134.9 \\
17.2\end{array}$ & $\begin{array}{l}2.19 \\
0.55\end{array}$ & $\begin{array}{r}174.8 \\
50.2\end{array}$ & $<116.1$ & $\begin{array}{l}41.5 \\
9.97\end{array}$ & $0920-150$ & 1090 & 1931 \\
\hline VLSS & J0918.1+2950 & $\begin{array}{rrr}09 & 1811.41 \\
& 0.31\end{array}$ & $\begin{array}{r}+295025.2 \\
5.1\end{array}$ & $\begin{array}{l}1.39 \\
0.16\end{array}$ & $<91.0$ & $<49.9$ & & $0900+250$ & 458 & 1731 \\
\hline VLSS & J0918.1-3010 & $\begin{array}{rrr}09 & 1811.50 \\
& 0.41\end{array}$ & $\begin{array}{r}-3010 \quad 17.5 \\
5.2\end{array}$ & $\begin{array}{l}1.72 \\
0.21\end{array}$ & $<83.4$ & $<73.8$ & & $0936-350$ & 1579 & 1707 \\
\hline VLSS & J0918.2+6451 & $\begin{array}{rr}09 & 1813.55 \\
& 0.51\end{array}$ & $\begin{array}{r}+645107.2 \\
3.6\end{array}$ & $\begin{array}{l}3.61 \\
0.40\end{array}$ & $\begin{array}{r}68.0 \\
7.2\end{array}$ & $<34.6$ & $\begin{array}{l}31.2 \\
4.83\end{array}$ & $0920+650$ & 1052 & 1004 \\
\hline VLSS & J0918.2+8111 & $\begin{array}{rr}09 & 18 \quad 13.88 \\
& 2.10\end{array}$ & $\begin{array}{r}+811127.8 \\
5.2\end{array}$ & $\begin{array}{l}1.40 \\
0.17\end{array}$ & $<71.9$ & $<58.4$ & & $0800+850$ & 602 & 550 \\
\hline VLSS & J0918.2-0053 & $\begin{array}{rr}09 & 18 \quad 14.01 \\
& 0.62\end{array}$ & $\begin{array}{r}-005346.6 \\
9.2\end{array}$ & $\begin{array}{l}0.86 \\
0.14\end{array}$ & $<139.5$ & $<76.2$ & & $0920-050$ & 1089 & 1615 \\
\hline VLSS & J0918.2-3048 & $\begin{array}{rr}09 & 1816.11 \\
& 0.25\end{array}$ & $\begin{array}{r}-304850.7 \\
3.4\end{array}$ & $\begin{array}{l}4.01 \\
0.42\end{array}$ & $<51.1$ & $<36.3$ & & $0936-350$ & 1573 & 1615 \\
\hline VLSS & J0918.2+6844 & $\begin{array}{rr}09 & 18 \quad 17.01 \\
& 0.83\end{array}$ & $\begin{array}{r}+684406.9 \\
4.6\end{array}$ & $\begin{array}{l}1.01 \\
0.12\end{array}$ & $<56.1$ & $<55.6$ & & $0920+650$ & 1047 & 1563 \\
\hline VLSS & J0918.3-0005 & $\begin{array}{r}091823.32 \\
0.67\end{array}$ & $\begin{array}{r}-000510.1 \\
6.7\end{array}$ & $\begin{array}{l}0.96 \\
0.15\end{array}$ & $<135.1$ & $<73.0$ & & $0920-050$ & 1083 & 1732 \\
\hline VLSS & J0918.4-2709 & $\begin{array}{rr}09 & 1824.02 \\
0.23\end{array}$ & $\begin{array}{rr}-2709 & 09.7 \\
3.2\end{array}$ & $\begin{array}{l}5.15 \\
0.55\end{array}$ & $\begin{array}{r}33.2 \\
7.4\end{array}$ & $<40.0$ & $\begin{array}{l}125.9 \\
26.12\end{array}$ & $0900-250$ & 436 & 705 \\
\hline VLSS & J0918.4-3133 & $\begin{array}{rr}09 & 1824.17 \\
0.32\end{array}$ & $\begin{array}{r}-313332.7 \\
3.7\end{array}$ & $\begin{array}{l}3.36 \\
0.43\end{array}$ & $\begin{array}{l}56.3 \\
11.4\end{array}$ & $<46.1$ & $\begin{array}{r}86.5 \\
11.90\end{array}$ & $0936-350$ & 1564 & 1508 \\
\hline VLSS & J0918.4+7143 & $\begin{array}{rr}091827.66 \\
1.82\end{array}$ & $\begin{array}{r}+714356.6 \\
7.7\end{array}$ & $\begin{array}{l}0.56 \\
0.09\end{array}$ & $<99.5$ & $<81.8$ & & $1000+750$ & 1491 & 596 \\
\hline
\end{tabular}

Note. - Description of columns:

1. Source Name (in the form "VLSS JHHMM.m+DDMM" as per International Astronomical Union (IAU) recommendations)

2. Right Ascension (J2000)

3. Declination (J2000)

4. Integrated Flux Density in Jy

5. Size of Deconvolved Major Axis in arcseconds, or the $2 \sigma$ upper limit if the source is unresolved

6. Size of Deconvolved Minor Axis in arcseconds, or the $2 \sigma$ upper limit if the source is unresolved

7. PA of Major axis in degrees.

8. VLSS Image Field

9. $\mathrm{X}$ pixel of source in VLSS Image Field

10. Y pixel of source in VLSS Image Field

Note. - Errors, if applicable, are given in the same units in the next row. 


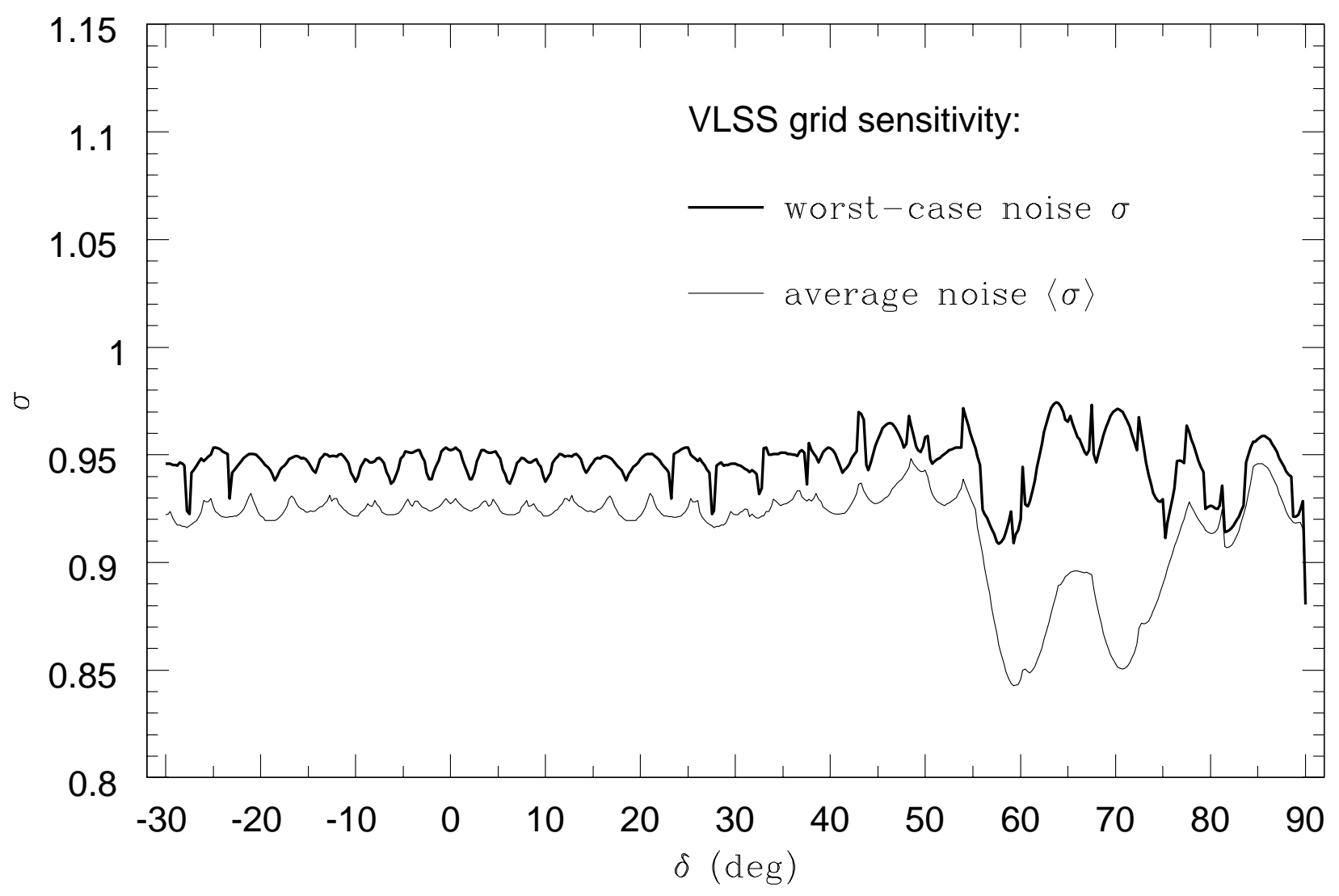

Fig. 1.- A plot showing the average (light line) and worst case (bold line) RMS noise (yaxis) as a function of declination (x-axis), based on our VLSS pointing grid and the shape of the $74 \mathrm{MHz}$ VLA primary beam. This assumes a constant RMS noise level for each field, with $\sigma=1$ normalized to the noise level at the center of any individual pointing. Due to the hexagonal observing pattern, the effects shown are not a simple function of declination. The worst case noise is only slightly higher than the average, indicating that the VLSS pointing grid produces essentially uniform sensitivity across the entire region surveyed. 


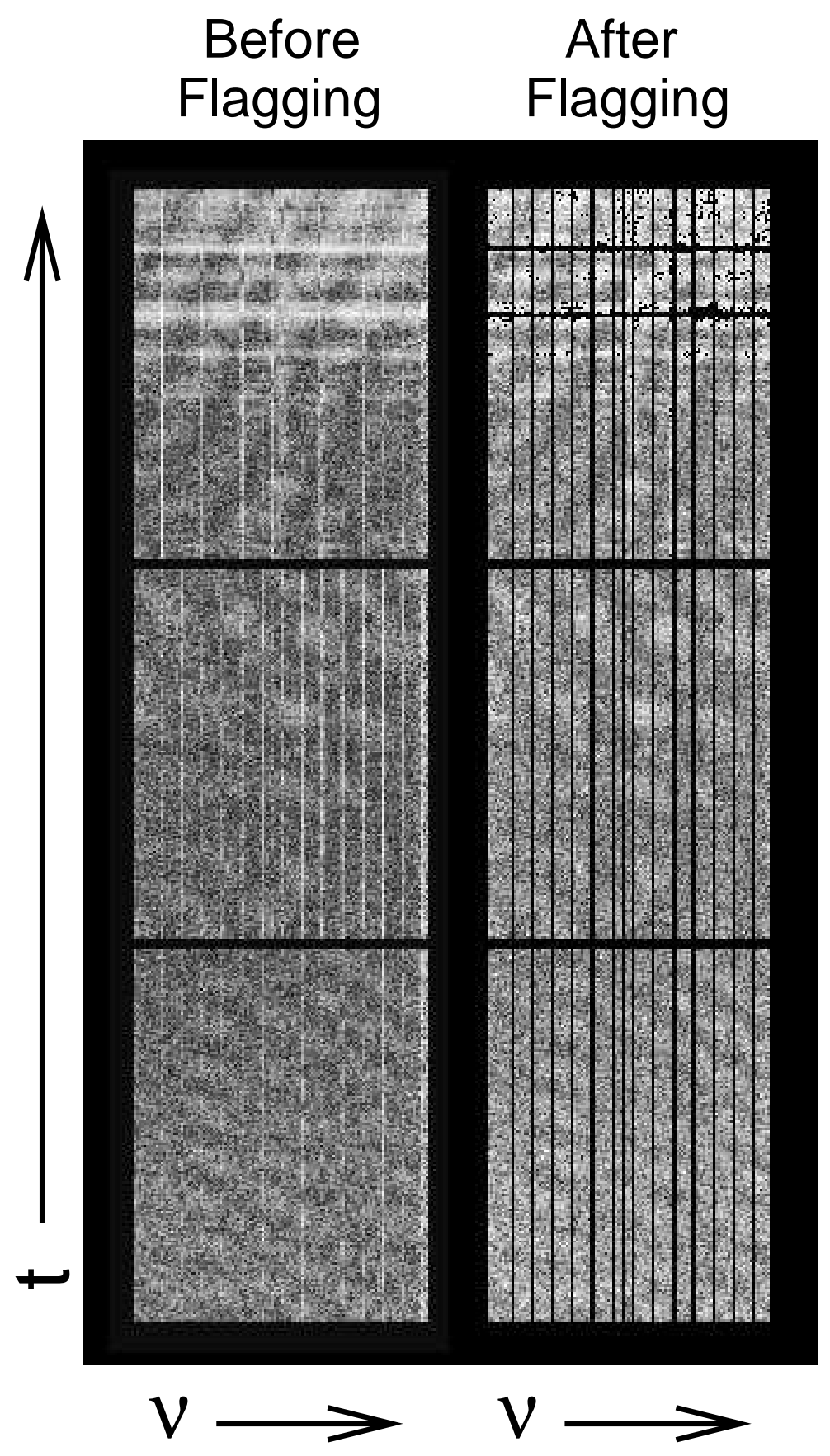

Fig. 2.- An illustration of the automated flagging procedure. Visibility amplitudes are shown for a single baseline in right circular polarization plotted with frequency channel along the horizontal axis and time interval along the vertical axis. The time is divided into three scans of roughly 25 minutes each. On the left are the data before flagging. Most of the smooth diagonal features are actual source structure. The entirely vertical features show channels in which RFI was present for long periods of time and the horizontal features show time ranges in which RFI was present across a wide range of frequencies. On the right are the data remaining after applying the automated flagging routines described in Section 4.2. Most of the worst RFI is successfully removed; however, some amount remains in particularly bad regions. 

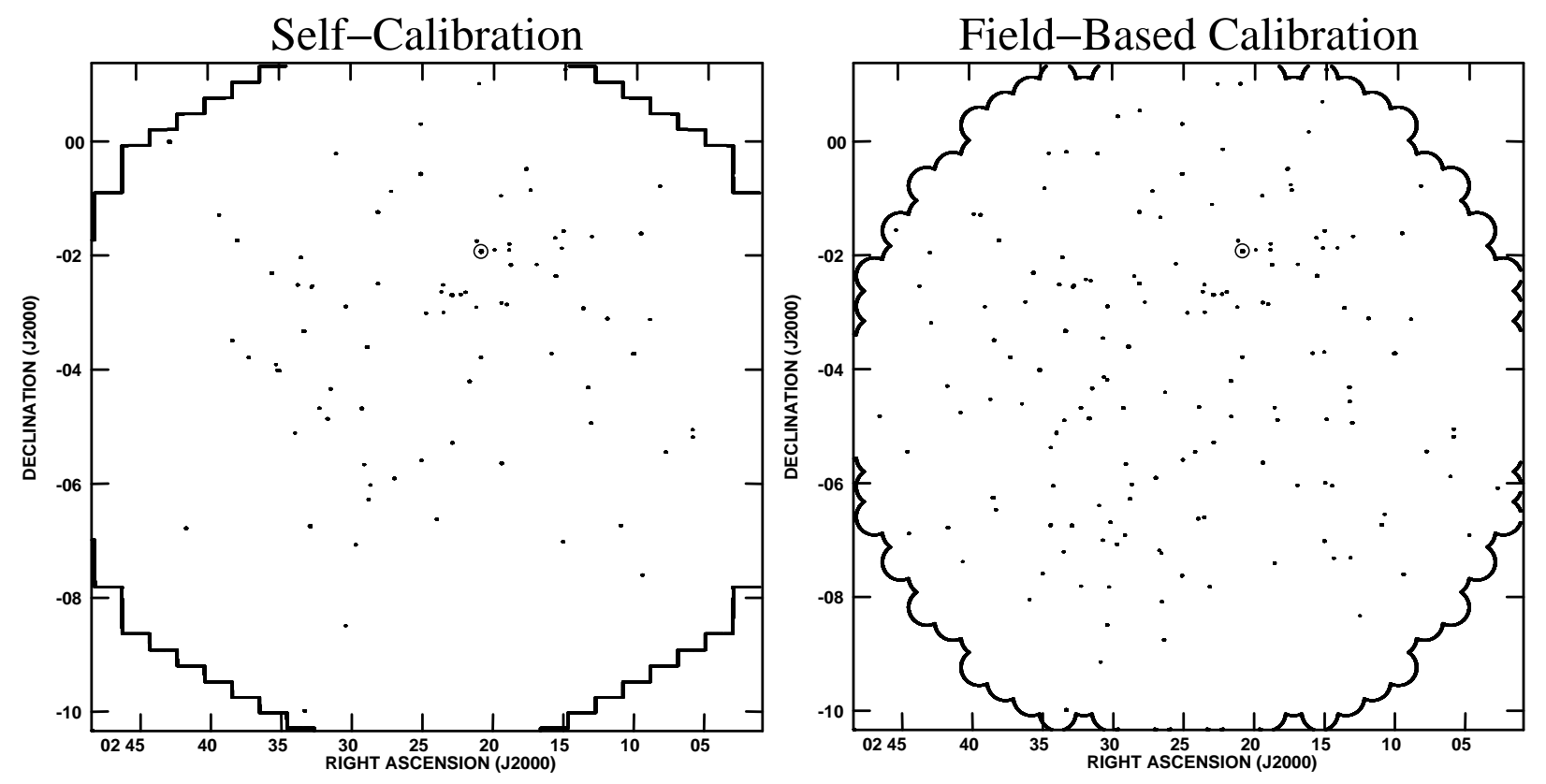

Fig. 3.- Comparison of self-calibration versus field-based calibration for the same $74 \mathrm{MHz}$ data set (Cohen et al. 2003). For each method, all sources with peak brightness above $400 \mathrm{mJy} /$ beam are plotted. The circled source is $3 \mathrm{C} 63$, which at $35 \mathrm{Jy}$ is much stronger than any other source in the field of view and therefore dominates the self-calibration. In the self-calibrated image, sources far from 3C 63 suffer increased ionospheric smearing due to increasingly un-correlated ionospheric phases. This causes the peak brightness of sources to decrease, lowering the apparent source density in the image. This problem is greatly improved in the field-based-calibrated image, which shows roughly uniform source density throughout the field of view. 

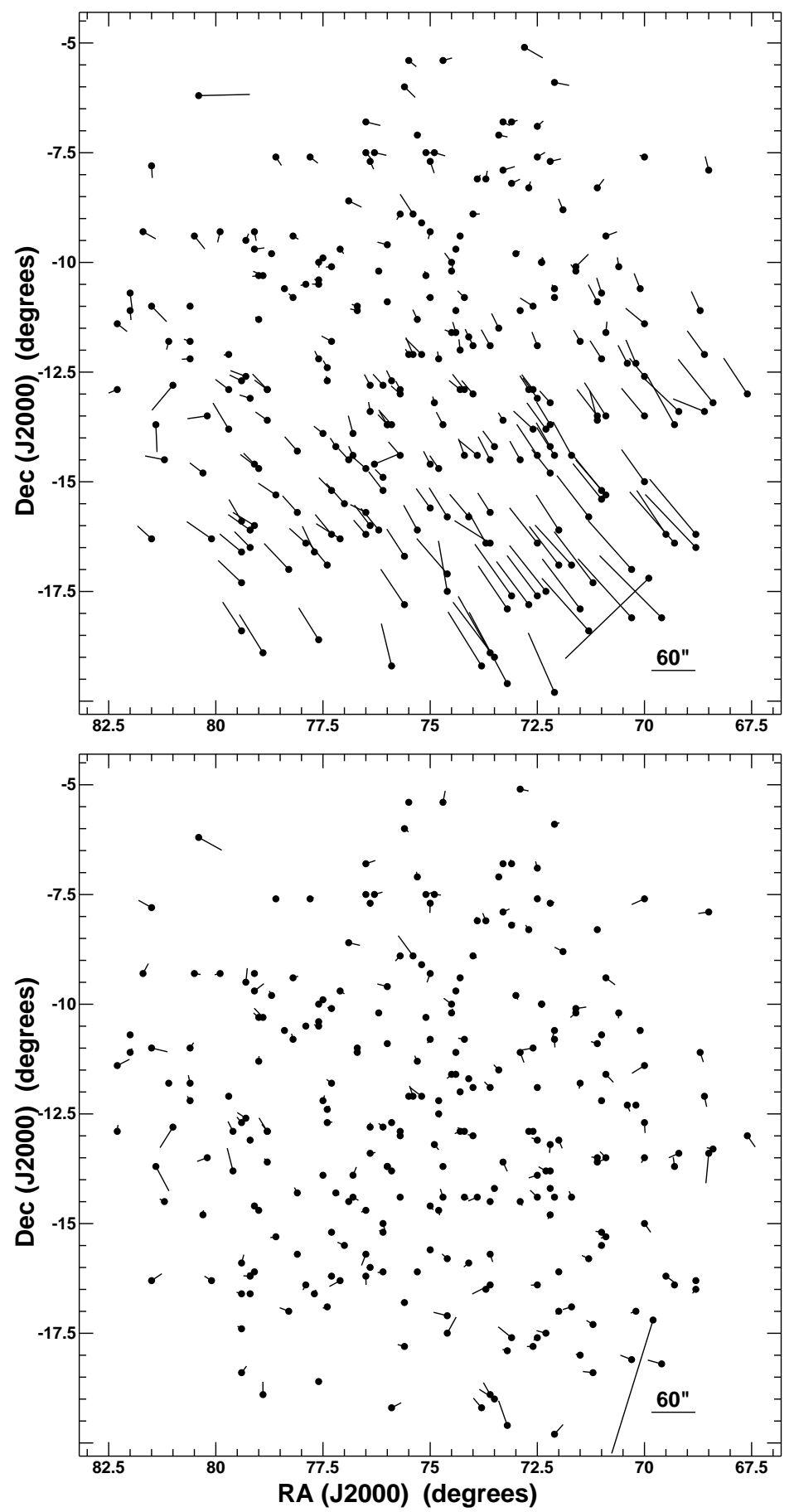

Fig. 4. - Source shifts relative to the NVSS positions for a VLSS field that had one of the worst cases of ionospheric calibration errors. The dots represent the VLSS source positions and the lines represent the distance and angle to the NVSS position. The shift magnitudes are magnified according to the scale shown at the lower right in order to be visible. The upper plot is before the corrections and the lower plot is after. Individual sources may be truly shifted from the NVSS positions due to actual differences in source centroids between 1400 and $74 \mathrm{MHz}$, however any true shifts should be random and not correlated with nearby sources. 


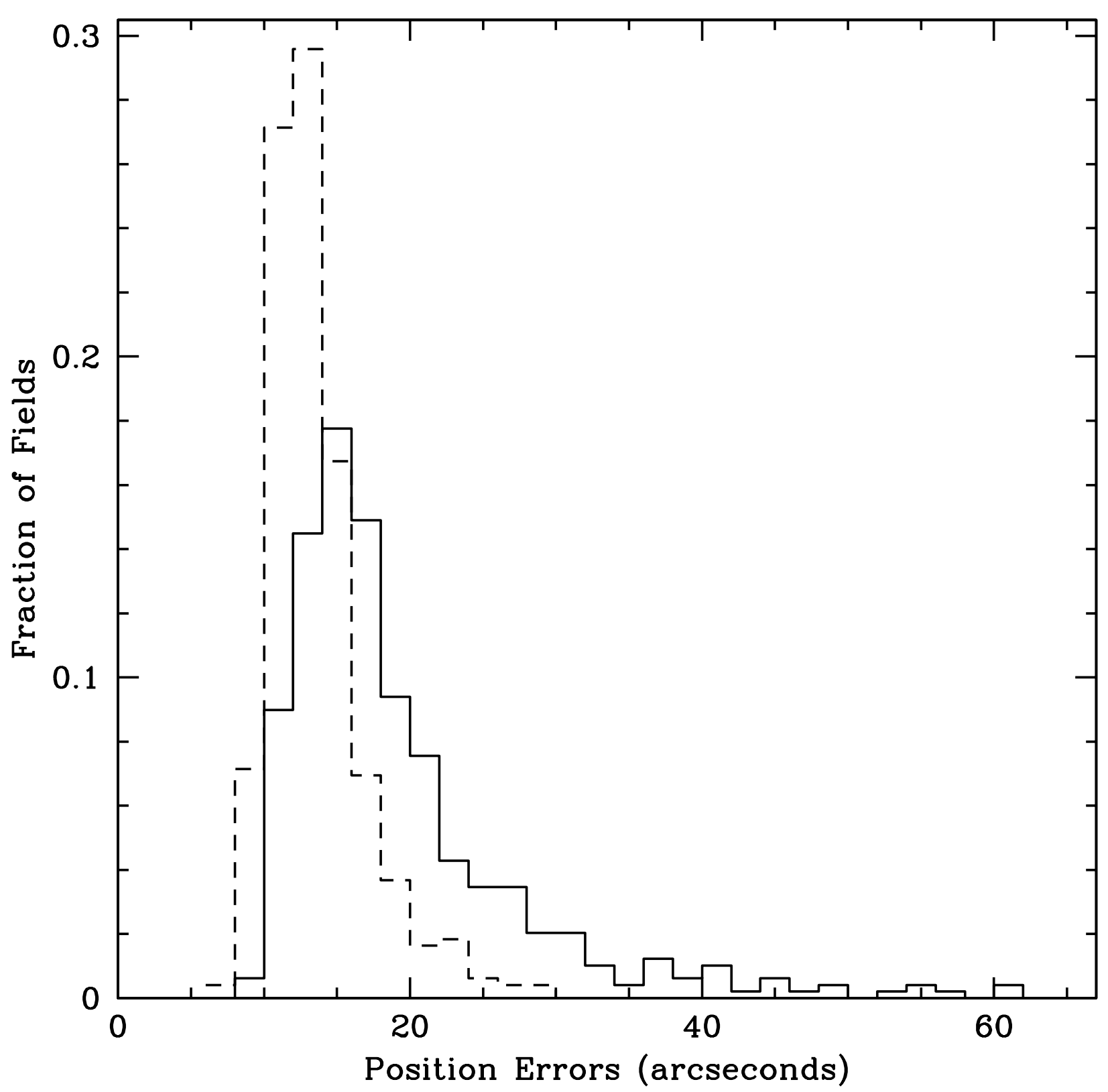

Fig. 5.- Histogram of position errors (for the 85th percentile of all sources) for all fields before correction (solid line) and after correction (dashed line). Before correction $10 \%$ of fields had position errors greater than $30^{\prime \prime}$, with some over $60^{\prime \prime}$. After correction, no field had position errors more than $30^{\prime \prime}$, and about $95 \%$ have position errors parameter less than $20^{\prime \prime}$, or $1 / 4$ of a beam width. 


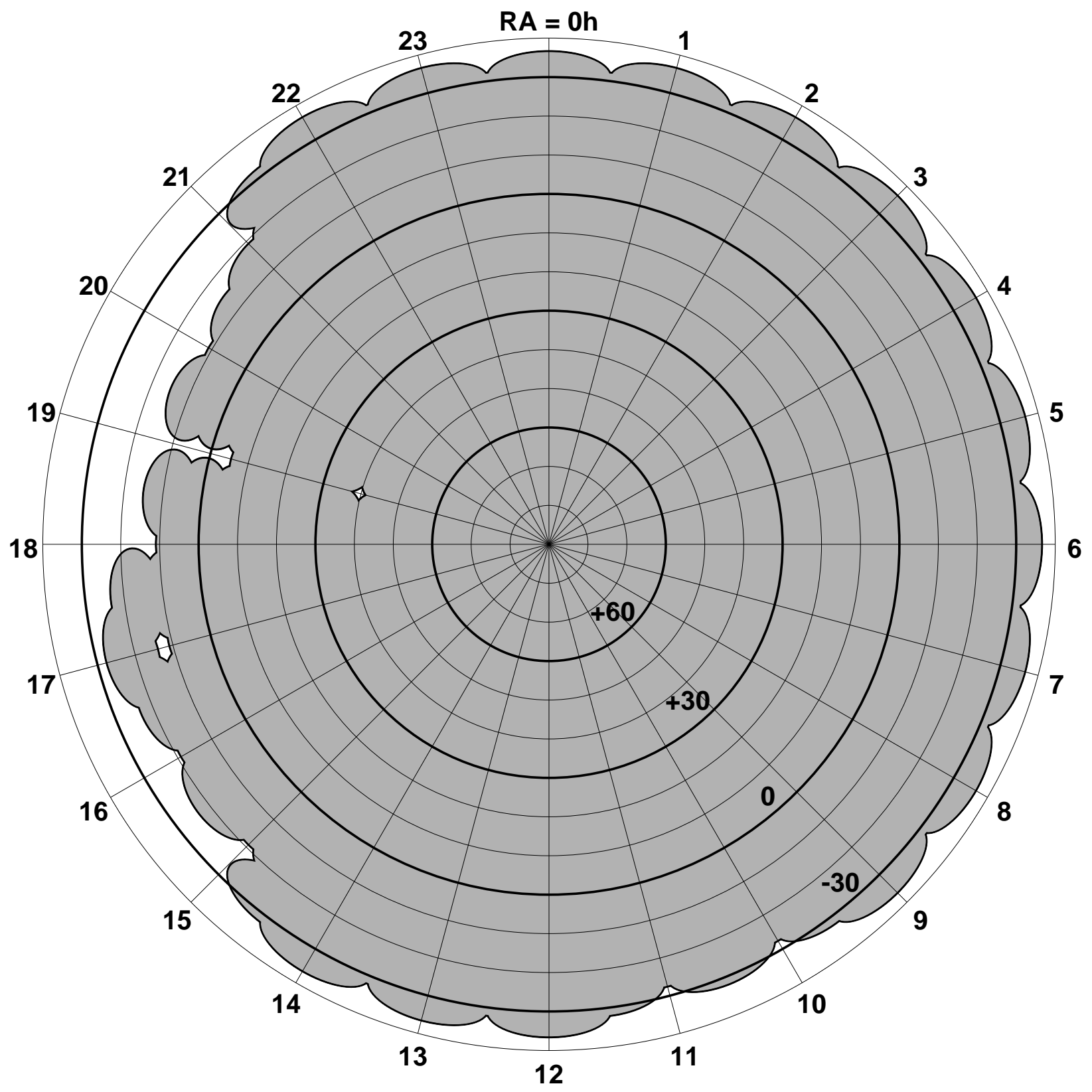

Fig. 6.- Region of sky currently imaged by the VLSS project. Currently about $95 \%$ of the sky above $\delta>-30^{\circ}$ is now covered, and further observations are planned to fully complete this region. 


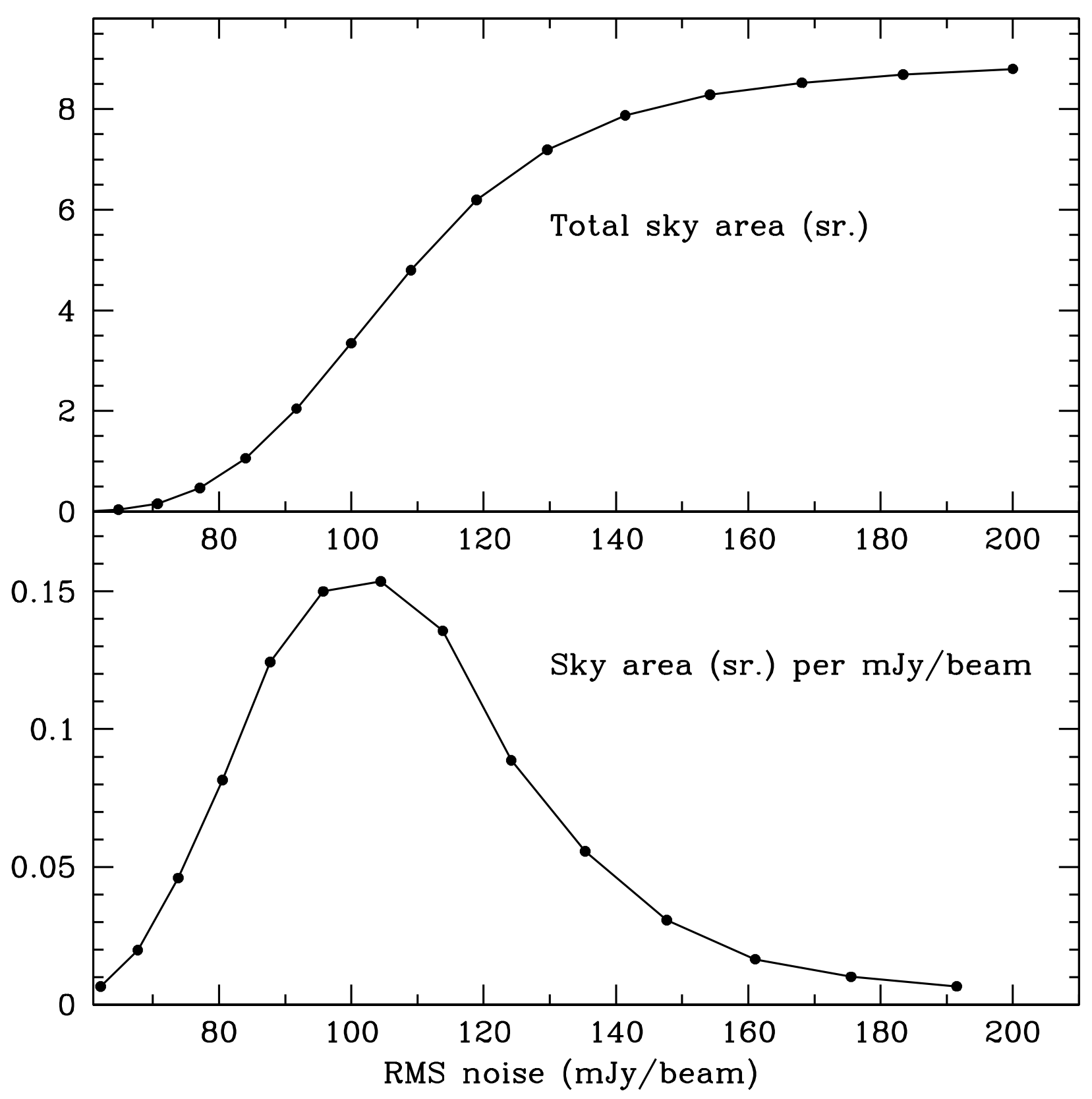

Fig. 7. - Top: Total sky area (y-axis) at or below a given RMS noise level (x-axis). Bottom: Differential sky area (y-axis) at a given RMS noise level (x-axis). The median RMS noise level is $108 \mathrm{mJy} /$ beam. The extended "tail" at higher noise levels is due mostly to regions around very bright sources (such as Cygnus A, Casseopeia A, Virgo A, etc.), regions of sky with high sky temperature like the Galactic plane, and regions at the edges of the mosaic maps which have no overlap with other fields. 

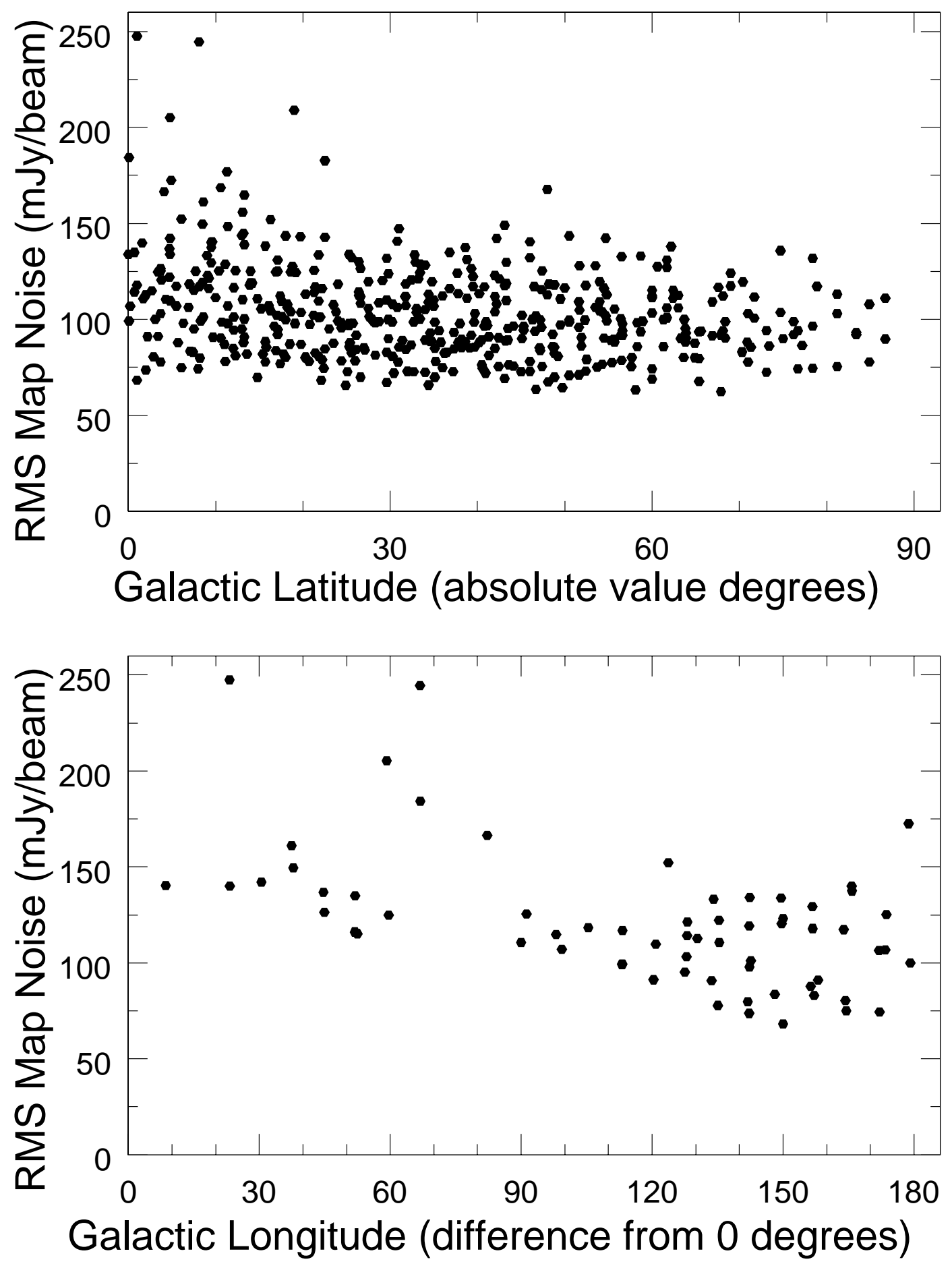

Fig. 8.- Top: RMS map noise for each field plotted against the absolute value of the Galactic latitude of the field center. Bottom: RMS map noise for each field located within $10^{\circ}$ of the Galactic plane plotted against the Galactic longitude of the field center in terms of degrees from the Galactic center. For both plots, fields located close to extremely strong sources such as Cygnus A were removed. 


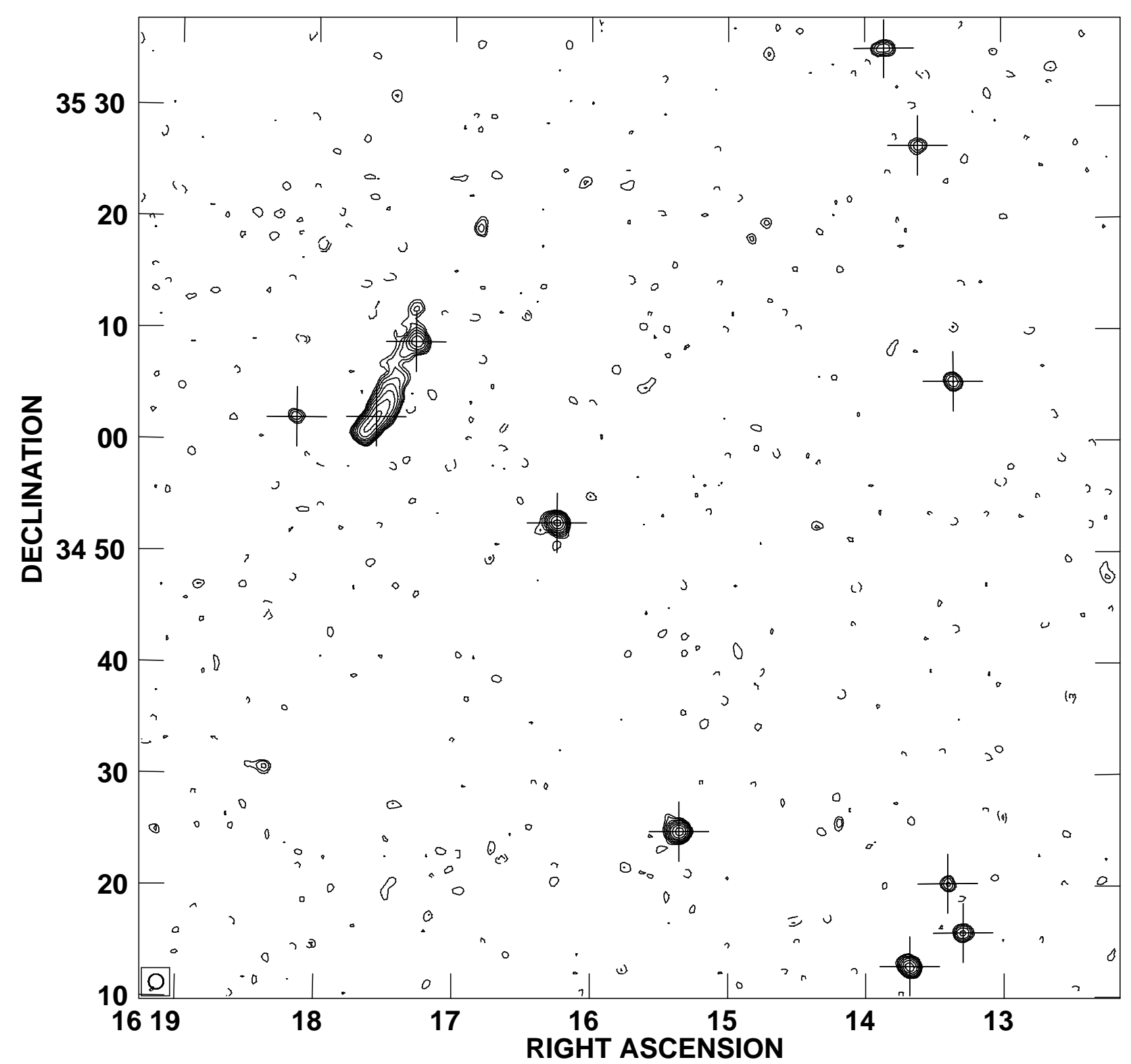

Fig. 9.- Contour plot of a sample sub-image from the VLSS. The peak flux density is 2.57 $\mathrm{Jy} /$ beam and the overall RMS noise level is $74.9 \mathrm{mJy} /$ beam. Contours begin at 2.5 times the RMS noise level (187.25 mJy/beam) and increase by factors of $\sqrt{2}$. The crosses indicate the locations of $5 \sigma$ source detections which are included in the source catalog. 

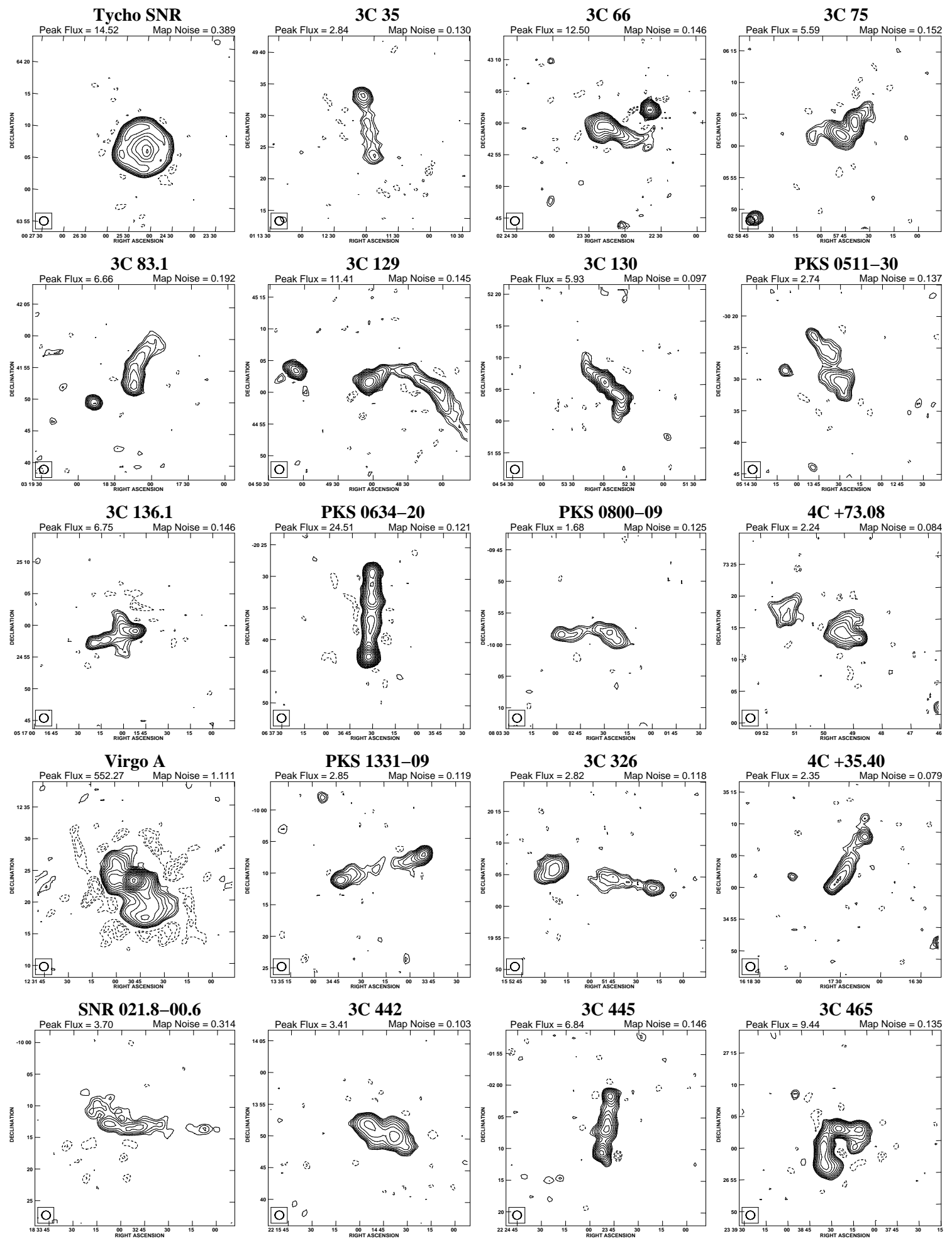

Fig. 10. - Contour plots of some of the larger sources observed by the VLSS. Peak flux density and map noise are given in Jy/beam. Contours begin at 2.5 times the map noise and increase by factors of $\sqrt{2}$. Sources are each labeled by their common radio names. 


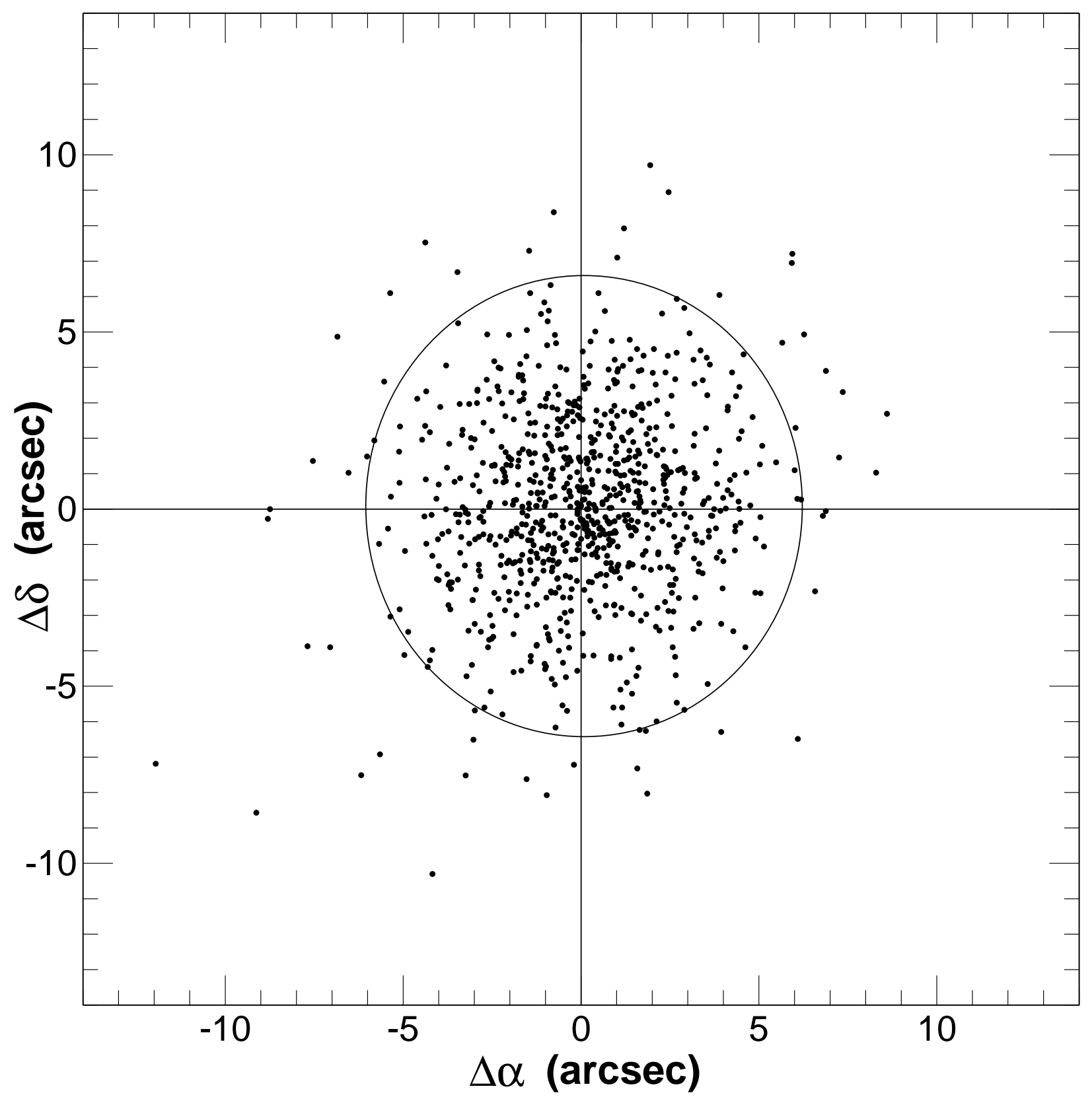

Fig. 11. - Source offsets of VLSS sources from their NVSS locations for the 866 sources strong enough that errors due to map noise are negligible, yet below the peak brightness threshold above which they might be used as calibrators. The ellipse represents the $90 \%$ confidence region based on the fitted mean and RMS of these offsets. 


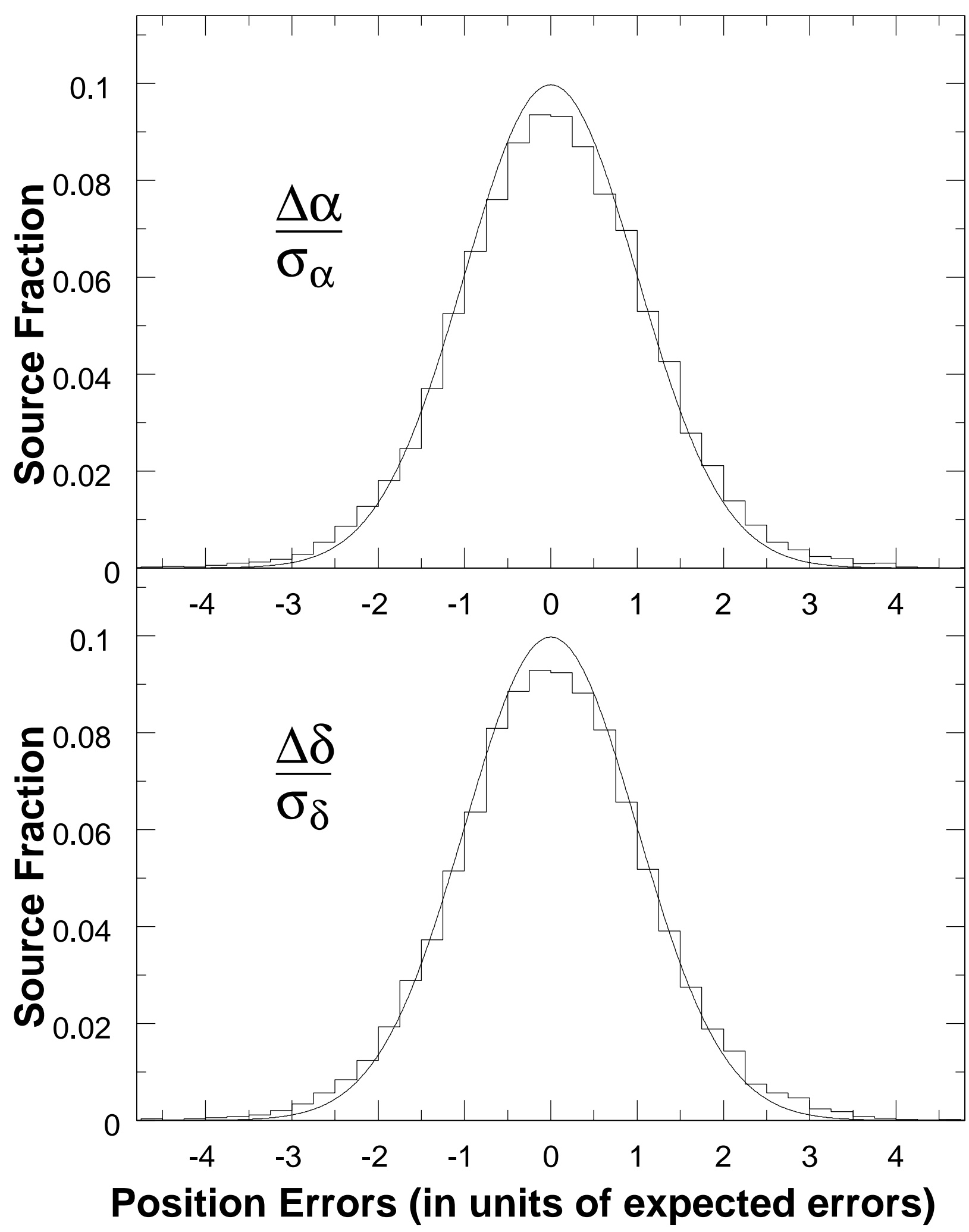

Fig. 12.- Histograms of the offsets in right ascension $(\Delta \alpha)$ and declination $(\Delta \delta)$ in units of the predicted position errors for each source. Only weak sources with peak flux less than $10 \sigma$ were included. The curves plotted over the histograms are normalized Gaussians with an RMS equal to 1, which is the theoretically expected distribution. 


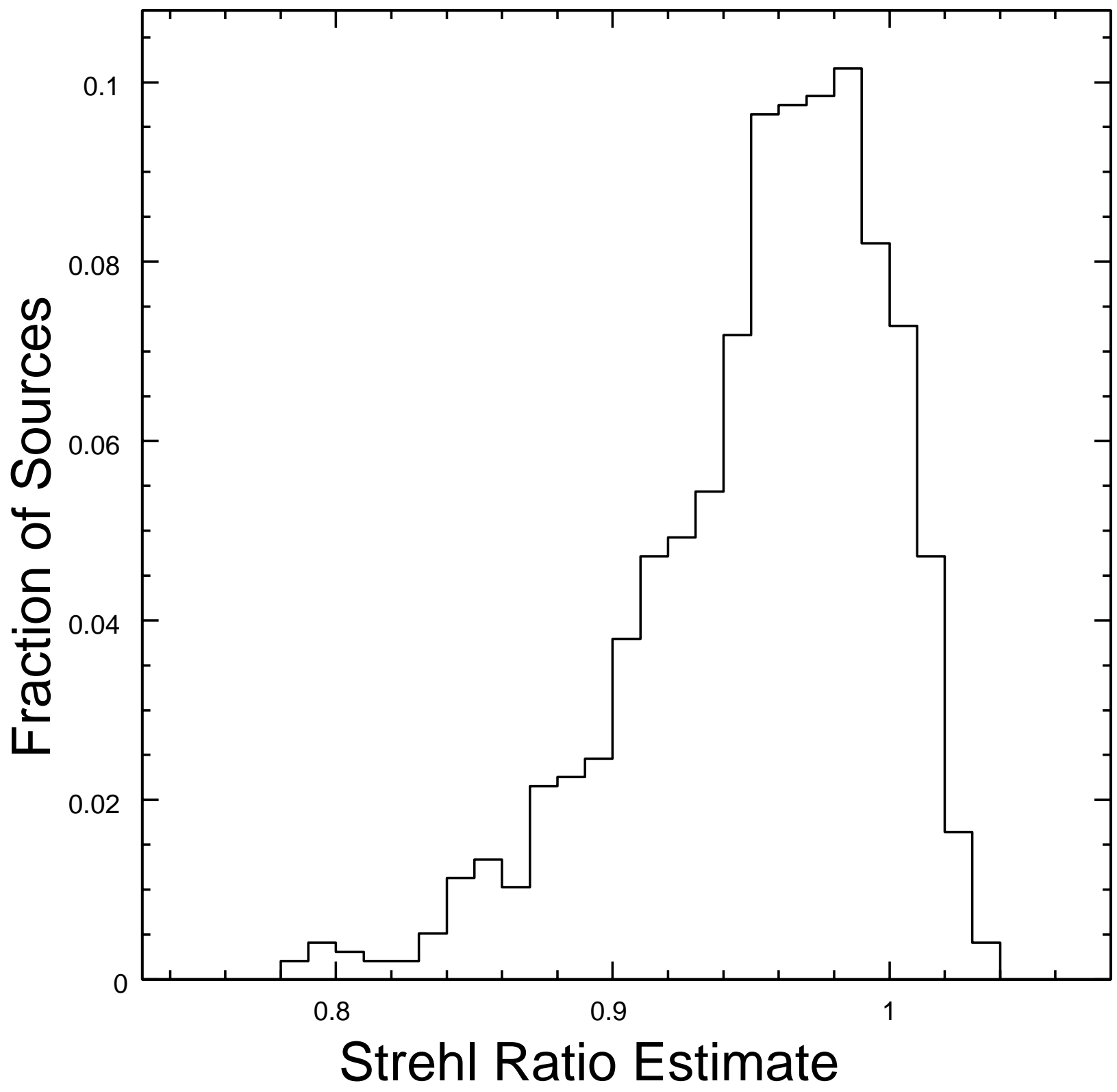

Fig. 13.- Distribution of estimated Strehl ratios for 975 bright, compact VLSS sources. The median Strehl ratio is 0.96 . There is a long tail of values well below the median, which could be due to observations with particularly high ionospheric calibration residuals. Equally plausible is that some fraction of sources have sizes that really do appear larger at $74 \mathrm{MHz}$ than at $1.4 \mathrm{GHz}$ because of diffuse steep spectrum emission. 


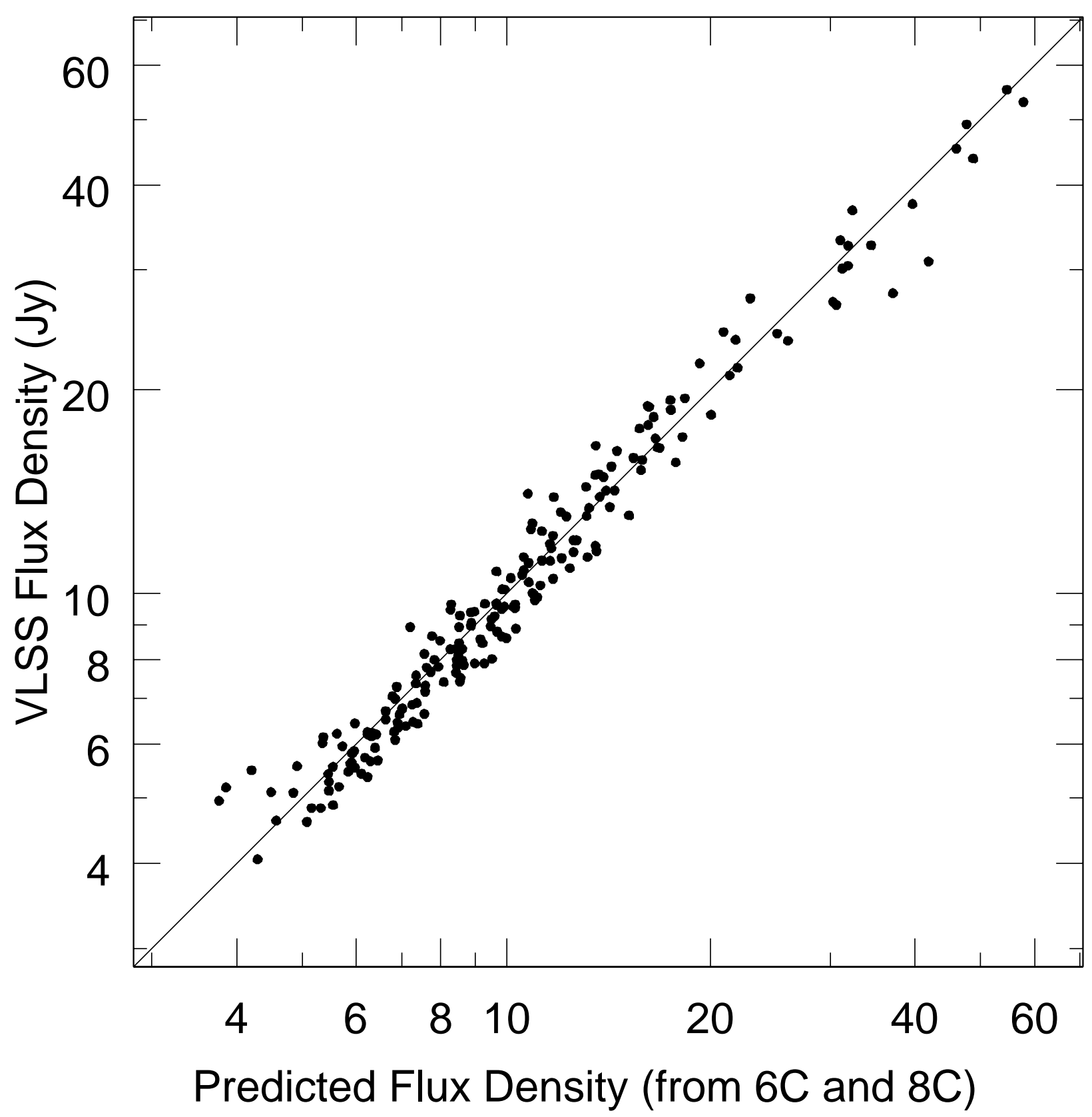

Fig. 14.- Comparisons of predicted flux densities versus measured flux densities for bright VLSS sources. Predicted flux densities at $73.8 \mathrm{MHz}$ were calculated by interpolating between the $6 \mathrm{C}$ and $8 \mathrm{C}$ flux densities for the 201 bright VLSS sources that had counterparts in both the $6 \mathrm{C}$ and $8 \mathrm{C}$ surveys. The average ratio of the VLSS flux densities to the predicted values was $0.99 \pm 0.01$ with a scatter of $\pm 10.4 \%$. The straight line represents locations for which the predicted and measured flux densities are equal. 


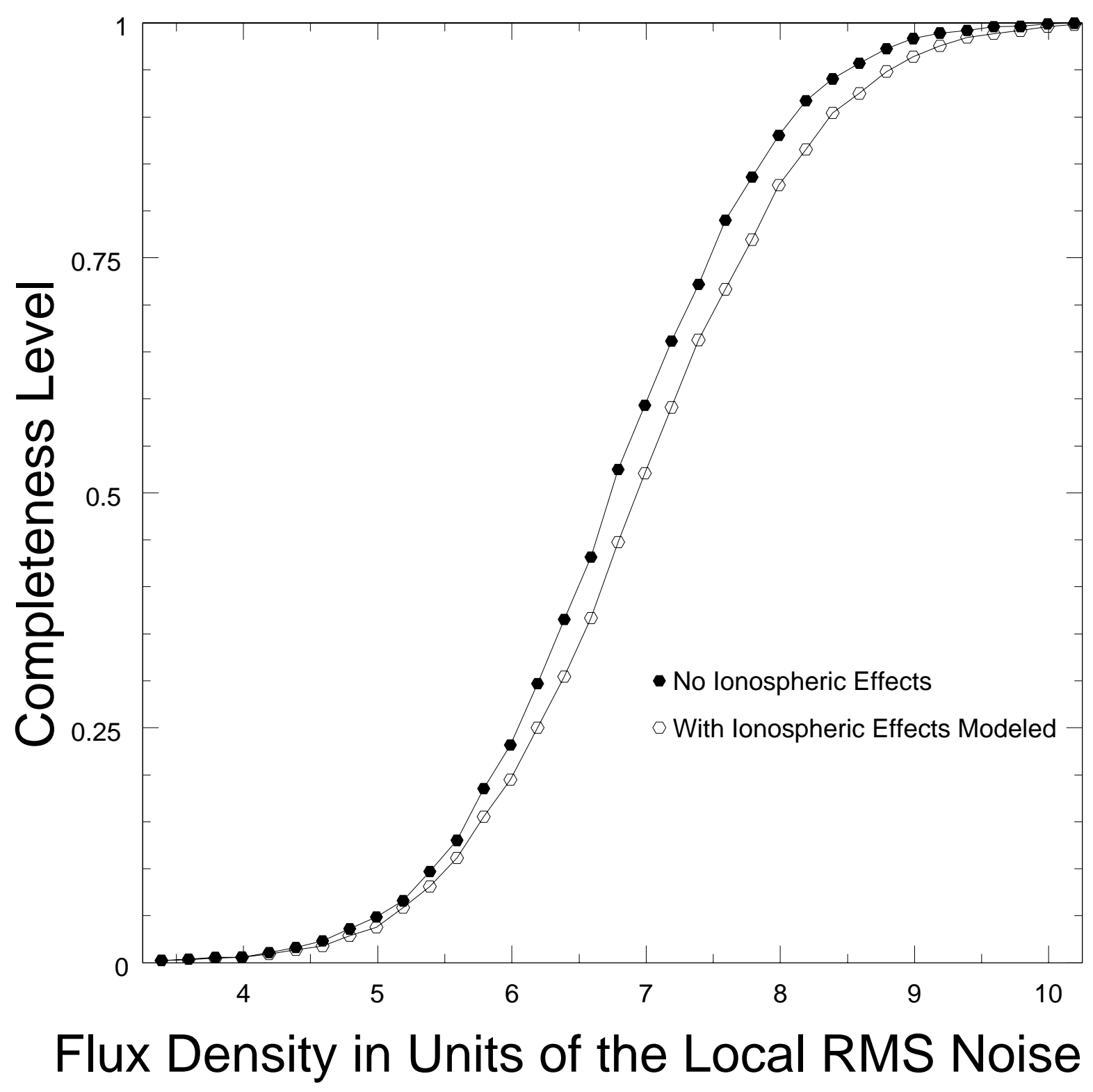

Fig. 15.- Differential completeness of the VLSS catalog for point sources as a function of the ratio of peak flux density to local RMS noise level. (This completeness measure does not apply to the regions surrounding the strongest sources for which higher source selection criteria were used.) The filled points are calculated from simulations that do not take ionospheric smearing into account, while the open points are from simulations that do account for this. The entire curve is shifted to the right by the clean bias which is 1.39 in units of the local RMS noise. 